This item was submitted to Loughborough's Research Repository by the author.

Items in Figshare are protected by copyright, with all rights reserved, unless otherwise indicated.

\title{
Numerical simulation of speckle noise in laser vibrometry
}

PLEASE CITE THE PUBLISHED VERSION

PUBLISHER

(C) Optical Society of America

LICENCE

CC BY-NC-ND 4.0

\section{REPOSITORY RECORD}

Rothberg, Steve. 2019. "Numerical Simulation of Speckle Noise in Laser Vibrometry". figshare. https://hdl.handle.net/2134/3314. 
This item was submitted to Loughborough's Institutional Repository by the author and is made available under the following Creative Commons Licence conditions.

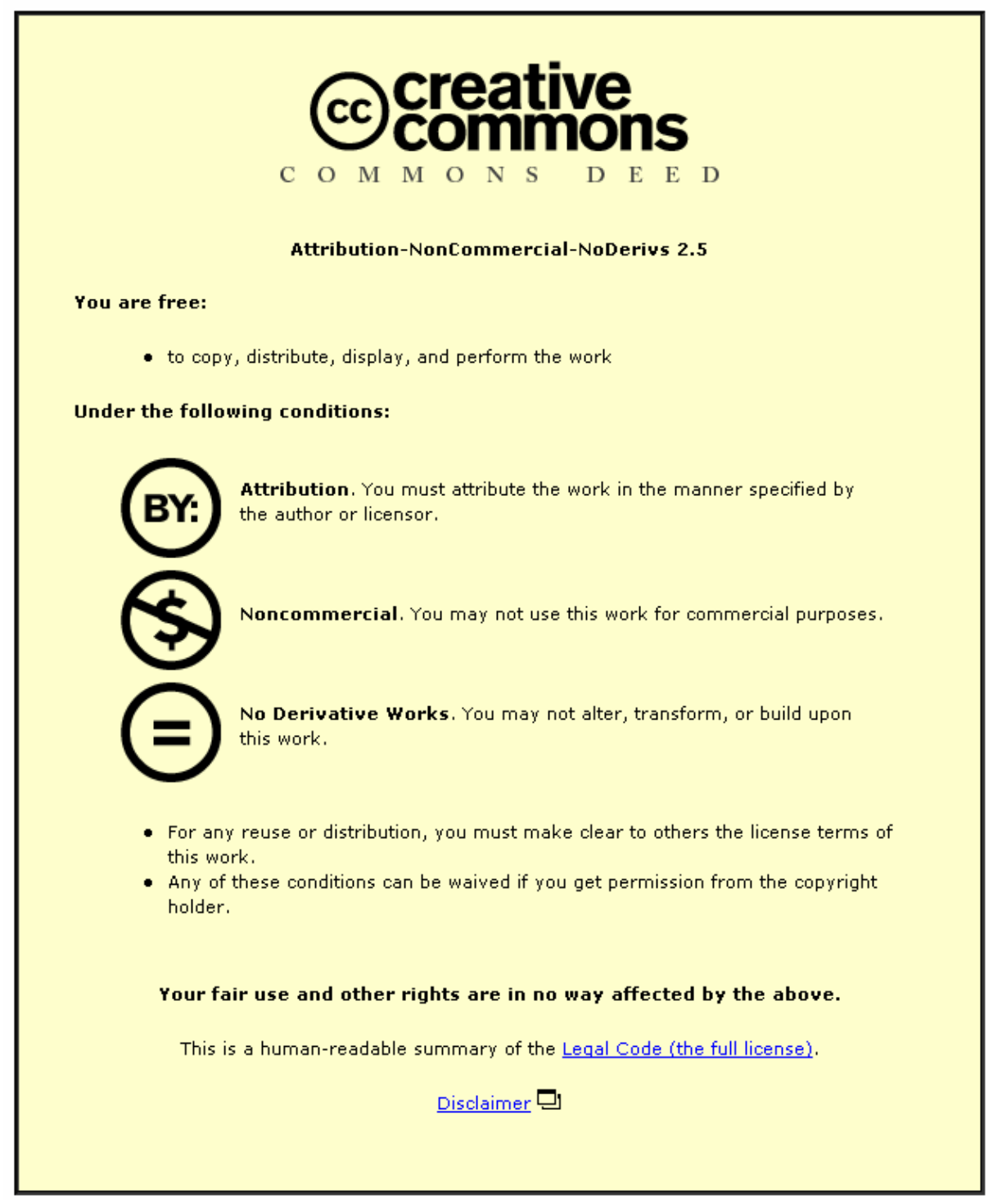

For the full text of this licence, please go to: http://creativecommons.org/licenses/by-nc-nd/2.5/ 
Numerical Simulation of Speckle Noise in Laser Vibrometry

\author{
Prof Steve Rothberg
}

Wolfson School of Mechanical and Manufacturing Engineering

Loughborough University, Loughborough, Leics LE11 3TU, UK 


\begin{abstract}
This paper considers the fundamental mechanism by which speckle noise is generated in Laser Vibrometry before describing a new numerical simulation for prediction of speckle noise level in a real measurement. Factors within the simulation include rate of change of phase within individual speckle transitions, low pass filtering to match the frequency range of experimental data with which comparison is to be made, a track-and-hold facility for periods of low signal amplitude and wavefront curvature effects. The simulation data provides real insight into the phase and amplitude modulation of the Doppler signal and good agreement is found in the final comparison with experimental data from a measurement on a rotating target.
\end{abstract}

OCIS codes:

120.7280 Vibration analysis, 030.6140 Speckle 


\section{Introduction}

When a coherent laser beam is incident on a surface that is optically rough, i.e. the surface roughness is large on the scale of the laser wavelength and the laser beam size exceeds any lateral scale to the surface roughness, the component wavelets of the scattered light become dephased. This condition is satisfied by almost all of the surfaces likely to be encountered in engineering structures. The dephased, but still coherent, wavelets interfere constructively and destructively, thus resulting in a chaotic distribution in backscatter of high and low intensities, referred to as a “speckle pattern”.

The phenomenon of laser speckle was first reported by researchers working with the very earliest continuous wave lasers in the early 1960s. They talked about a "remarkable granular or peppery nature not present in ordinary light”", "a sparkling appearance”, and, less dramatically, "random dark and light spots”3. Much early work on laser speckle, described as “Enemy Number One” by Gabor ${ }^{4}$, was directed at eliminating its detrimental effect in holography until techniques such as Laser Speckle Photography ${ }^{5,6}$ began to show how the speckle effect could be used to advantage in metrology. Since the early 1970s, applications in so-called 'Speckle Metrology ${ }^{77}$ have been numerous.

The development of Laser Vibrometry, for non-contact vibration measurements from solid surfaces, has its origins in Laser Doppler Anemometry (LDA), a technique for non-intrusive measurements in fluid flows first reported in $1964^{8}$. Measurements in fluids require the flow to be seeded in order to ensure sufficient intensity of scattered light but vibration measurements on solid surfaces encounter no such difficulties and this application of the technique was recognised 
at an early stage. It is only in the last 15 years, however, that vibration measurements using Laser Vibrometers have started to become commonplace. Users tend to be vibration engineers rather than optical specialists and applications have tended to be challenging. As a consequence of both these factors, speckle effects have begun to establish themselves as the crucial influence on Vibrometer performance.

Laser Vibrometers are interferometric devices, typically incorporating a frequency shift in the reference beam in order to discriminate the direction of target motion ${ }^{9}$. They differ principally by the method used to produce the reference beam frequency shift and also in the interferometric arrangement used. During the 1970s, a variety of different devices were reported incorporating frequency shifting by a rotating diffraction grating ${ }^{10}$, by Bragg cell ${ }^{11}$ and by rotating scattering $\operatorname{disc}^{12}$. It was in the design incorporating the scattering disc that the effects of the speckle phenomenon first became apparent in Laser Vibrometry. This design went on to become one of the first commercial instruments, manufactured by Brüel \& Kjær, with a noise floor dominated by the effect of the changing speckle pattern generated by the rotating scattering disc.

While this design of Vibrometer has now been superseded, the range of likely applications has now increased to include a variety of measurements directly from rotating structures such as magnetic discs ${ }^{13,14}$, bladed discs ${ }^{15,16}$, tyres and other automotive components ${ }^{17}$, spindles $^{18}$ and modal analysis on rotating discs ${ }^{19}$ and it is in such measurements that the effects of laser speckle are most apparent and most problematic. These applications used single beam Vibrometers which are suitable for translational vibration measurement. Rotational vibration measurements torsional, pitch, yaw and roll vibrations - require parallel beam arrangements ${ }^{20,21}$ and these 
'Rotational Laser Vibrometers' are also now available commercially. Measurements are not limited to rotating structures but they are routinely used on rotors for applications including assessment of torsional damper health ${ }^{22}$ or crankshaft bending vibration ${ }^{23}$. Since measurements with these devices are so likely to be made on rotating structures, users have found the influence of laser speckle to be particularly undesirable.

This paper begins with a review of the fundamental mechanism by which speckle noise is generated in Laser Vibrometry before describing a numerical simulation of speckle behaviour for prediction of noise level in a real measurement. The intention of the simulation is not to re-create the complexity encountered in a real speckle pattern but to 'simplify' the speckle pattern and concentrate on the key aspects of the noise generating mechanisms.

Figure 1 shows a schematic diagram of the interferometer used to gather experimental data. The rotating diffraction grating is used to divide the laser beam and to introduce a frequency shift between the two resulting beams. The lens makes the beams parallel for convenience and a large polarising beam splitter directs the beams to either target or reference surfaces. The quarter-wave plate ensures that light initially reflected at the beamsplitter is transmitted on scattering from the surfaces, after which the mirror / beamsplitter arrangement re-combines them on the photodetector. Components are positioned such that optical paths are exactly matched. Where consideration of the effect of collecting increasing numbers of speckles on the photodetector is made, this is conveniently achieved in experimentation simply by moving the photodetector closer to the second beamsplitter. In this way, coherence between each beam in the interferometer is easily maintained. 


\section{Laser Speckle and Pseudo-Vibration}

Figures $2 \mathrm{a} \& \mathrm{~b}$ show, respectively, the form of the speckle patterns generated by scattering from a diffuse surface and by scattering from the retro-reflective tape that is often used with Laser Vibrometers as surface treatment. The speckle pattern produced by scattering from retroreflective tape has a very distinctive Airy disc form as a result of diffraction at the glass beads on the surface of the tape. Statistically the speckle phases are uniformly distributed between $-\pi$ and $\pi$ and, neglecting the intensity distribution of the Airy disc, speckle intensities have a negative exponential probability distribution ${ }^{24}$.

In the interferometer, the detected intensity, $I$, is given by the time-average of the square of the total light amplitude resulting from combination of target and reference beams. In its standard form this is written ${ }^{25}$ as

$$
I=\bar{I}+\Delta I=\left(I_{R}+I_{T}\right)+2 \sqrt{I_{R} I_{T}} \cos \left[\left(2 \pi f_{R} t-2 k a(t)\right)+\left(\phi_{R}-\phi_{T}\right)\right]
$$

where $I_{R}$ and $I_{T}$ are the reference beam and target beam intensities respectively, $\phi_{R}$ is the reference beam phase across the detector and $f_{R}$ is its frequency shift, $\phi_{T}$ is the target beam phase at the detector if the target surface were stationary, $k$ is the light wavenumber and $a(t)$ is the target vibration displacement.

The intensity sum, $\bar{I}=\left(I_{R}+I_{T}\right)$, is of no value in the vibration measurement and is usually filtered in some way. The second and relatively higher frequency term is generally referred to as 
the "Doppler signal" and is the component which, on demodulation, yields the time derivative of the target vibration displacement i.e. vibration velocity. The frequency of the Doppler signal is known as the "beat frequency", $f_{\text {beat }}$, and is given by the modulus of the time derivative of the cosine argument,

$$
f_{\text {beat }}=\left|f_{R}-\frac{2}{\lambda} \frac{d a(t)}{d t}\right|=\left|f_{R}-f_{D}\right|
$$

where $f_{D}$ is the Doppler shift in the target beam. Equation (2) shows that the beat occurs at the frequency difference between the two beams. Frequency demodulation of the photodetector signal by an appropriate Doppler signal processor produces a time-resolved voltage analogue of the target vibration velocity.

In reality, at least one of the beams incident on the photodetector takes the form shown in figure 2 with the photodetector sampling a small region within the speckle pattern. The Doppler signal, as described in equation (1), is therefore the result of a summation of speckles. This is usually of little practical consequence unless the speckles start to move or evolve in response to target motions. When this does happen, there are two important effects on the Doppler signal. The first is an amplitude modulation and the second is a phase modulation, both of which result from the summation on the photodetector being performed over a changing population of speckles. Following equation (1), the Doppler signal can be written as

$$
\Delta I=I_{\text {res }}(t) \cos \left[\left(2 \pi f_{R} t-2 k a(t)\right)+\Phi_{\text {res }}(t)\right]
$$


where $I_{\text {res }}$ and $\Phi_{\text {res }}$ are the time-varying resultant amplitude and phase of the Doppler signal. A Doppler signal described in this way was the basis of an analytical study ${ }^{26}$ of uncertainty in Laser Vibrometers including the effect of thresholding as implemented in the track-and-hold systems discussed later in this paper.

The amplitude modulation gives Doppler signals the characteristic appearance shown in figure 3. It is possible for the Doppler signal amplitude to drop to a very low level in which case signal drop-outs can occur and glitches appear in the Laser Vibrometer output, contributing significant broadband noise. Often the importance of amplitude modulation is over-emphasised, however, when considering the effects of laser speckle. Generally, the signal amplitude will remain at an acceptable level and phase modulation will be the major concern. The phase modulation cannot be seen easily in figure 3 but it is certainly present and its manifestation in the Laser Vibrometer output is very clear. Figure 4 shows the form of the Laser Vibrometer output from a "measurement" on a non-vibrating, rotating target. This is "speckle noise”. Figures 5a\&b show typical experimental data comparing speckle noise (low-pass filtered at $20 \mathrm{kHz}$ ) and the normalised Doppler signal RMS. The figures show the same data presented in two different ways. Figure 5a shows, in particular, how speckle noise increases in this experiment at low target-detector separations where Doppler signal RMS is especially high, emphasising how speckle noise is not a simple function of Doppler signal amplitude. Figure 5b presents these same data in terms of numbers of speckles collected as this format is more helpful to the discussion of the numerical simulation to be presented in this paper. Note that the critical parameter is not the total number of speckles collected (i.e. the ratio of detector size to speckle size) but the ratio of detector size in the direction of speckle motion to the speckle size. A square photodetector was 
used so Doppler signal RMS increases approximately in direct proportion to the square root of the total number of speckles collected, as expected.

With the Doppler signal phase now a function of time, the beat frequency expression given originally in equation (2) takes a new form with an additional term,

$$
f_{\text {beat }}=\left|f_{R}-\frac{2}{\lambda} \frac{d a(t)}{d t}+\frac{1}{2 \pi} \frac{d \Phi_{r e s}(t)}{d t}\right|
$$

The frequency content of $\left(d \Phi_{\text {res }} / d t\right)$ will obviously appear in the output spectrum and this is worthy of further consideration. If the speckle pattern changes are induced by non-normal target motions, such as tilt, in-plane motion or rotation, which, in general, will be periodic with the same fundamental frequency as the on-axis vibration, then $\left(d \Phi_{\text {res }} / d t\right)$ will be pseudo-random in nature with the same fundamental frequency as the on-axis vibration. The periodic nature of the speckle noise is apparent in figure 4, which relates to a "measurement" on a rotating, nonvibrating target, in which the added circles are intended to aid the reader in seeing the periodicity. The characteristic spectrum of a pseudo-random signal consists of approximately equal amplitude peaks at the fundamental frequency and higher order harmonics. This additional signal content, first described as pseudo-vibration ${ }^{25}$, will be indistinguishable from the genuine vibration information and the spectrum of the data in figure 4 is shown in figure 6 . The strength of the harmonic peaks serves to emphasise the periodicity of the output signal and a typical characteristic of the speckle noise generated in measurements on rotating targets is the way that the amplitudes of the harmonic peaks are maintained up to very high frequencies. Figure 6 shows peaks up to $12 \mathrm{x}$ fundamental frequency but the speckle peaks in this data maintained their amplitude well above 100x fundamental frequency. For targets with less rapid speckle 
transitions, such as those with only in-plane motion, the speckle peaks are more likely to decrease within the first few harmonics, giving a spectrum with a similar appearance to that from a harmonic vibration with a small amount of harmonic distortion. In each case, the speckle noise is concentrated at the frequencies in which the vibration engineer is likely to be most interested and a certain degree of judgement is required when interpreting low level data obtained with a Laser Vibrometer. Whenever speckle transitions do occur, the speckle noise generated will dominate any other noise source in the instrument output and the impressively low noise-floors quoted by manufacturers will not be attainable.

\section{Numerical Simulation}

\section{Review of Previous Work}

The intensity and phase of the Doppler signal resulting when $P$ target beam speckles, with phase $\phi_{T p}$, intensity $I_{T p}$ and area $A_{p}$ on the photodetector (total area $A$ ) are mixed with a uniform reference beam has been shown to be $\mathrm{e}^{25}$

$$
\begin{gathered}
I_{\text {res }}=\frac{2}{A}\left[I_{R} \sum_{p=1}^{P} \sum_{q=1}^{P} A_{p} A_{q} \sqrt{I_{T p} I_{T q}} \cos \left(\phi_{T p}-\phi_{T q}\right)\right]^{1 / 2}, \\
\tan \Phi_{\text {res }}=\left[\left(\sum_{p=1}^{P} A_{p} \sqrt{I_{p}} \sin \left(\phi_{T p}-\phi_{R}\right)\right) /\left(\sum_{p=1}^{P} A_{p} \sqrt{I_{p}} \cos \left(\phi_{T p}-\phi_{R}\right)\right)\right] .
\end{gathered}
$$

These expressions were at the heart of early simulations performed ${ }^{27}$ and they remain the basis of the numerical simulation to be described in this paper. For more general cases, for example where the reference beam contribution is itself a speckle pattern, it is convenient to work with 
the quadrature components of each contribution to the Doppler signal and this is how the summations are performed within the numerical simulation. With $N$ reference beam speckles, each with phase $\phi_{R n}$, intensity $I_{R n}$ and area $A_{n p}$ overlapping the $P$ th target beam speckle and incident on the photodetector, the summations take the following forms:

$$
\begin{gathered}
I_{\text {res }}=\frac{1}{A}\left[\left[\sum_{n=1}^{N} \sum_{p=1}^{P} A_{n p} \sqrt{I_{R n} I_{T p}} \sin \left(\phi_{R n}-\phi_{T p}\right)\right]^{2}+\left[\sum_{n=1}^{N} \sum_{p=1}^{P} A_{n p} \sqrt{I_{R n} I_{T p}} \cos \left(\phi_{R n}-\phi_{T p}\right)\right]^{2}\right]^{1 / 2}, \\
\tan \Phi_{\text {res }}=\left[\left(\sum_{n=1}^{N} \sum_{p=1}^{P} A_{n p} \sqrt{I_{R n} I_{T p}} \sin \left(\phi_{R n}-\phi_{T p}\right)\right) /\left(\sum_{n=1}^{N} \sum_{p=1}^{P} A_{n p} \sqrt{I_{R n} I_{T p}} \cos \left(\phi_{R n}-\phi_{T p}\right)\right)\right] .
\end{gathered}
$$

\section{Phase Changes During Speckle Transitions}

Early simulations examined the phase change resulting from whole speckle transitions across a photodetector. For the case of a single speckle on the photodetector that is entirely replaced by a second uncorrelated speckle, it is easy to show how the random distribution of speckle phases leads to a mean phase change over many transitions of zero and a variance of $\left(\pi^{2} / 3\right) \mathrm{rad}^{2}$. For more than one speckle, the mathematical complexity increases significantly and numerical simulation is necessary. The early simulations performed agreed with the theoretical prediction for the single speckle case and showed how the variance decreased with increasing ratio of detector size in the direction of speckle motion to speckle size, $M$, according to the empirical relationship $^{27}$

$$
\operatorname{Var}\left[\Delta \Phi_{\text {res }}(t)\right]=\frac{\pi^{2}}{3 M^{0.85}}
$$


Figure 7 shows how the variance initially decreases rapidly, by a factor of about 4 up to $M=5$. For larger $M$, the variance continues to decrease but more slowly with values down to around $0.2 \operatorname{rad}^{2}$ for configurations of practical interest. Combination of phase change data from the simulation with correlation times for dynamic laser speckles ${ }^{28,29}$ gave the first speckle noise predictions but these failed to give good agreement with experimental levels ${ }^{27}$.

One reason for this was that initial work had concentrated on the phase change in the Doppler signal during a single speckle transition and implicitly assumed that the rate of change of phase was constant throughout the transition. That this is not the case is obvious from figure 8 which shows how phase varies through the fading-out of a sinusoidal component (with phase arbitrarily set at -1 ) and the fading-in of a sinusoidal component (with phase arbitrarily set at +2 ). The maximum rate of change is significantly underestimated by using the phase change across the full transition. In the numerical model this, therefore, introduces the need to sub-divide each speckle transition but the number of sub-divisions required is a matter for consideration.

The full single speckle transition is now considered as $W$ partial transitions each of equal duration and incurring a phase change, $\Delta \Phi^{\prime}$. To proceed, a value of $W$ must be found beyond which the rate of change of phase within each partial transition can be considered constant. At this point, $\operatorname{Var}\left[W \Delta \Phi^{\prime}\right]$, which can also be written $W^{2} \operatorname{Var}\left[\Delta \Phi^{\prime}\right]$, becomes constant. The simulation showed that $W=20$ represented a suitable compromise between the time taken to run the simulation and achieving the criterion on variance, as shown in figure 9 . In the simulations presented later, $W=40$ was used. Figure 9 also shows how the true variance in the phase change 
is a factor of around 4 higher than is obtained by calculating on the basis of the full transitions and this has a consequent influence on the accuracy of the prediction.

\section{Effects of Wavefront Curvature}

For the arrangement shown in figure 1, simple consideration of the phase change due to speckle transitions, as described in the previous section and indicated in figure 7 , would lead to the conclusion that minimum noise will result when a maximum number of speckles are collected i.e. at the closest target-detector separation possible. This is not borne out in figures $5 \mathrm{a} \& \mathrm{~b}$, however, where speckle noise increases significantly at the higher values of $M$, closer to the target. This is the result of wavefront curvature. In terms of speckle behaviour, the speckles (which are long and thin) move in to and then out of the plane of the detector as they translate across its surface. The distance travelled is particularly significant at small target-detector separations. For this reason, the simulation breaks each speckle into a $W \mathrm{x} H$ grid, typically $40 x 10$, in order to apply a curvature correction to the detected speckle phase.

\section{Structure of the Numerical Simulation}

The simulation has been written to model the speckle noise resulting when the target laser beam is incident in a radial direction on the circumference of a rotating (nominally circular crosssection) shaft as shown in figure 1 . The simulation opens by receiving optical data (wavelength, beam diameter, radius of curvature at the target), configuration data (photodetector size), target data (radius and rotation speed), 'measurement' data (upper frequency set for the FFT) and data specifically for the simulation ( $W, H$ and the number of full speckle transitions). From this 
information, target-detector separations are chosen at which the ratio of detector size in the direction of speckle motion to speckle size takes integer values. The number of speckle transitions is also adjusted to ensure that the number of data points generated in the simulation is a power of 2 for optimum FFT efficiency. A typical value in excess of 3200 (around 128000 partial speckle transitions) was used in the simulation. In terms of the experimental arrangement used, this is approximately equivalent to the speckle transitions occurring during half a rotation cycle.

Creation of the 'target' speckle pattern matrix is then initiated. Figure 10 is intended to help visualise the physical interpretation of the matrix generated. The figure shows, in heavy dashed lines, the fixed position of a photodetector whose dimensions are, in this case, 2 speckles by 2 speckles. The speckles have a regular shape - the principal simplification used in the simulation - and translate across the photodetector surface. In the existing simulation only speckle translation, rather than boiling ${ }^{28}$, is considered as this is reasonable for the configuration under scrutiny. Each speckle has intensity with a negative exponential probability density generated from a random number, $0 \leq x_{p} \leq 1$, as follows,

$$
\frac{I_{T p}}{\langle I\rangle}=-\ln \left(1-\frac{x_{p}}{1+(1 \mathrm{e}-10)}\right),
$$

where $\langle I\rangle$ is the mean speckle intensity and the quantity (1e-10) is added to the denominator of the $\ln$ argument to prevent an attempt to evaluate $\ln (0)$ while limiting distortion of the probability distribution. Speckles are assigned phases in the range $-\pi$ to $\pi$ using a sequence of random 
numbers independent of that used to generate intensity values. The 'width' of the matrix, i.e. in the direction of speckle motion, is determined by the number of full speckle transitions over which the simulation is to be performed. The 'height' of the matrix is set by the maximum number of speckles to be collected in this dimension in any simulation. The detector should be regarded as having fixed physical size in all simulations and so speckle intensities are increased appropriately as $M$ increases through the simulation range.

For the purposes of the later inclusion of wavefront curvature and to ensure accuracy in the rate of change of phase calculation, each speckle is then divided into a $W x H$ grid. This also conveniently allows disruption of what would otherwise be a very regular presentation of each 'speckle' to the detector without attempting to replicate the full irregularity associated with speckle shapes. The variation in the position of the speckle on the detector, as shown in figure 10, is limited to $H$ steps.

The reference beam contribution can be either of uniform intensity or as a stationary speckle pattern. If the latter, then the arrangement of speckles on the detector is also disrupted. In this case, the rows (rather than the columns) of the speckle matrix are misaligned ( $W$ randomly selected possible positions) and the columns are set so that partial speckles are collected at the top and bottom of the detector.

Once the intensity and phase matrices are complete for the target and reference beam contributions, the simulation moves into its outer loop which controls the number of speckles incident on the photodetector for each completion of the inner loop of the simulation. In the 
simulation, values of $M$ from 1 to 20 were considered. Experimentally, this is achieved by varying the target-detector separation in the configuration of figure 1 between $10 \mathrm{~cm}$ and $1.2 \mathrm{~m}$. Corrections to speckle phase for wavefront curvature are also calculated once target-detector separation is fixed, ready for application within the inner loop.

The inner loop calculates the Doppler signal quadrature components after every partial speckle transition. At this stage, the target and reference beam contributions on the detector are in the form of matrices with dimensions $(M W)$ by $(\alpha M H)$ where $\alpha$ is the aspect ratio of the photodetector. The products $\sqrt{I_{R} I_{T}} \cos \left(\phi_{R}-\phi_{T}\right)$ and $\sqrt{I_{R} I_{T}} \sin \left(\phi_{R}-\phi_{T}\right)$ are first calculated for the corresponding elements in these matrices, before summation of each component across the whole detector. The quadrature components are used to provide Doppler signal amplitude and phase for each speckle summation and, from this, change in phase follows straightforwardly.

The following discussion concentrates on features of the phase change data since this is of most importance. At this stage, the phase change data is dominated by $2 \pi$ wraparounds, as shown in figure 11. These are artefacts of the simulation and result from the calculation of phase from the quadrature components. They are corrected by running the phase change matrix through a loop to detect phase changes in excess of $\pi$ which is the maximum change possible in a single transition. The resulting phase change data is shown in figure 12 in which smaller but still significant transient phase changes remain evident. Such a feature in the phase change data will have a significant effect on the speckle noise level across a broad frequency band. Further investigation reveals how these are related to periods of low signal amplitude, as also shown in figure 12 for two arbitrarily selected peaks in the phase change data. This finding is consistent 
with a statistical study ${ }^{30}$ which found that weighting the phase derivative by signal amplitude reduced the variance in the frequency estimate. In fact, the signal amplitude need only be low relative to its mean level rather than in an absolute sense for this to occur since increasing all the speckle intensities by some factor (e.g. by increasing laser power) will have no effect on the phase calculation. Laser Vibrometers sometimes use track-and-hold type circuits to contend with periods of low signal amplitude but, unless set with reference to an appropriate mean signal level, these are unlikely to be effective in limiting this noise generating mechanism. Nonetheless, the simulation also incorporates a track-and-hold facility so that the effect on speckle noise can be observed.

The data manipulation so far has been in terms of phase change but the speckle noise prediction requires rate of change of phase so the time taken for the partial transition, $T_{w}$, is required ${ }^{31}$. For the case of a laser beam incident with $\exp [-2]$ diameter $D$ and radius of curvature $r$ on a target of radius $R$ and rotation angular frequency $\Omega$ producing speckle with size $\left\langle\sigma_{0}\right\rangle$ on a photodetector at distance $z$ from the target,

$$
\begin{gathered}
T_{w}=\frac{1}{W \Omega}\left[2\left(\frac{R}{D}\right)^{2}+\left(\frac{R(1+z / r)+2 z}{\left\langle\sigma_{0}\right\rangle}\right)^{2}\right]^{-1 / 2}, \\
\left\langle\sigma_{0}\right\rangle=\frac{2 \sqrt{2}}{\pi} \frac{\lambda z}{D} .
\end{gathered}
$$

It is at this stage that the connection must be made between the simulation and the physical environment. Selection of an appropriate photodetector size fixes the speckle size for a given M, 
which in turn sets the distance, $z$. The time taken for speckle transitions is typically very short. In supporting experimentation in which target rotation was at $30 \mathrm{~Hz}$, equation (9a) indicates that the time for a full speckle transition would be in the region of $2 \mu$ s for the configuration to be discussed shortly. As a result, the speckle noise generated extends across an extremely broad frequency range, certainly well beyond the frequency range of general interest to a vibration engineer making a measurement of this kind. It is, therefore, essential to include a filter in the simulation set to the upper limit in the corresponding experimental analysis. The simulation proceeds by calculating the FFT of the speckle noise so that the noise measured in any chosen frequency range can be calculated.

\section{Results of the Simulation}

Figure 13 shows typical results from the simulation for the case of a uniform intensity reference beam contribution and in the absence of correction to the target beam speckle pattern for wavefront curvature. The trend demonstrated in figure 7 causes a reduction in speckle noise with increasing $M$ in the simulated data, both in total level and after low pass filtering at $5 \mathrm{kHz}$. In experimental data, such as in figure 5b, the opposite trend is observed at higher values of $M$ (close target-detector separations) and wavefront curvature in the target beam speckle pattern is responsible for this as demonstrated by figures $14 \mathrm{a} \& \mathrm{~b}$.

Figures $14 \mathrm{a} \& \mathrm{~b}$ show typical results from the simulation for the cases of a uniform intensity reference beam contribution and a speckled reference beam contribution respectively with adjustments made in both cases for wavefront curvature. For the uniform intensity case in figure 
14a, the total speckle noise has an RMS value in the range $60-90 \mathrm{~mm} / \mathrm{s}$. Such a high noise level would be prohibitive for a real measurement but recall that this is across a much broader frequency range than that of interest in this type of application. In the filtered data (upper frequency limit again $5 \mathrm{kHz}$ ), a considerable reduction in RMS speckle noise occurs, down to 4$7 \mathrm{~mm} / \mathrm{s}$ which is tolerable for such applications. By comparison with figure 13, the data shows how, at close target-detector separations such that $M \geq 3$, the effect of wavefront curvature is to increase significantly the variance of the phase change associated with speckle transitions leading to higher speckle noise levels. For $M \leq 2$, the trend follows that of the data in figure 13 . The track-and-hold facility has little effect except at the lowest values of $M$ where the few relatively low intensity target beam speckles collected produce lower Doppler signal amplitudes. This is because the uniform reference beam gives a strong Doppler signal whose amplitude rarely drops below the pre-set threshold. In the context of a real measurement, the simulation indicates that there is not a large variation in measured speckle noise after low-pass filtering at $5 \mathrm{kHz}$ but the data does show a minimum in the region of $M$ between 2 and 4.

In figure $14 \mathrm{~b}$ for the speckled reference beam, a 30\% higher noise level is encountered because of the increased presence of rapid changes in phase after periods of relatively low signal amplitude. The track-and-hold facility now has a dramatic effect on speckle noise amplitude because the additional noise is closely associated with low signal. Although the RMS speckle noise predicted is comparable with that from the uniform reference beam, the data quality will be degraded in a real measurement because of the number of track-and-hold interventions. For example, at $M=3$, the track-and-hold facility is invoked for $3.5 \%$ of the data length while at $M=1$, where the predicted level is relatively very low, the track-and-hold facility is invoked for $70 \%$ of 
the data length and a measurement would be very unsatisfactory. Within the $0-5 \mathrm{kHz}$ range, RMS levels between $5-8 \mathrm{~mm} / \mathrm{s}$ are predicted, in excess of those from the uniform intensity reference beam with a minimum value found in a similar region to the previous data.

\section{Comparisons with Experimental Data}

Figure 15 shows comparison of predicted and experimental Doppler signal amplitude, normalised because different laser beam intensities were used during experimentation and because the scaling of laser beam intensity is arbitrary as far as the simulation is concerned. The solid lines show the simulated trends, with the amplitude of the uniform intensity reference beam configuration predicted to be some ten times higher than that for the speckled reference beam. This is in acceptable agreement with experimental data which shows similar relative values and trends with the exception that the simulation does not accommodate the photodetector output saturation evident in the uniform intensity reference beam data points at the shorter targetdetector separations (higher values of $M$ ). For the speckled reference beam, the simulation predicts more rapid growth in amplitude at higher values of $M$. This will be considered in later developments of the simulation but it is not felt to be too significant to the speckle noise prediction because this is relatively unaffected by signal amplitude in this range.

Figures 16 and 17 show comparisons of predicted and experimental speckle noise amplitudes. Figure 16 shows data for the case of a uniform intensity reference beam contribution. Experimental data is shown as the mean of five measurements, with a small correction applied for the output noise from the Doppler signal processor in the absence of speckle transitions. 
Good agreement is found in the absolute values and in trends including the minimum in the range of $M$ between 2 and 4 . The figure shows a hint of how speckle noise increases at close target-detector separations (higher values of $M$ ) but the initial configuration in figure 1 did not allow such measurements so a second series of experiments with a suitably modified optical arrangement were performed to enable comparison. These results are shown in figure 17, this time for the case of the speckled reference beam. The higher values in this data are due to the effect of low-pass filtering at $20 \mathrm{kHz}$, rather than at $5 \mathrm{kHz}$ as in figure 16 , as a means to demonstrate further the good agreement found with the simulation. The experimental speckle noise levels are the same as those in figure 5b. Good agreement is again demonstrated in the absolute values and in trends including the minimum in the range of $M$ between 2 and 4 . Such good agreement is especially pleasing considering the complexity present in the combination of two independent speckle patterns from which the experimental data is generated. The simulation over-estimates the measured speckle noise levels by $10-20 \%$, believed to be the result of the abrupt nature of the transitions between its regularly shaped 'speckles'. In the real speckle patterns, irregularity in speckle shape smoothes these transitions, resulting in lower speckle noise levels. By including simulated data without the influence of wavefront curvature, figure 17 also emphasises the importance of the way in which speckles effectively move in to and out of the plane of the photodetector as they move across its surface. Noise levels at higher values of $M$ (close target-detector separations) appear dominated by this mechanism.

\section{Conclusions}


This paper has given details of a how speckle noise manifests itself in Laser Vibrometers together with a numerical simulation to enable explanation of the underlying phenomena in speckle noise generation and ultimately prediction of measured levels. The particular application examined was that of a measurement on a rotating target since this represents a worst case scenario as far as speckle noise is concerned. Configurations with uniform intensity and speckled reference beam contributions were both considered in the simulations.

The simulations showed the fundamental mechanism to be the changing population of randomly phased speckles, causing variation in the resultant Doppler signal phase. Speckle transitions across the photodetector were considered in increments of 1/40th of speckle size to prevent an underestimation of rate of change of phase that would otherwise occur. In the summations, each speckle was considered sub-divided into a 40x10 grid to enable corrections to be applied to speckle phase for the effects of wavefront curvature which were shown to be extremely significant at higher values of $M$, the ratio of detector size in the direction of speckle motion to speckle size. The noise generated in speckle transitions was shown to extend across an extremely broad frequency range, much broader than is of interest to the engineer considering the application under scrutiny. A reduction of the order of a factor of 10 was found in the predicted RMS speckle noise after low-pass filtering at $5 \mathrm{kHz}$, a frequency equal to one-hundredth of the reciprocal of the speckle transition time in the simulations presented. A particularly important contribution to overall noise level was made by peaks in the phase change data resulting after periods of relatively low Doppler signal amplitude. These may not be eliminated by track-andhold circuitry because signal amplitude may not have been 'low' in an absolute sense but 'low' relative to the mean signal level for a particular configuration. 
In final comparisons, good agreement was found between predicted and measured Doppler signal amplitude and predicted and measured speckle noise levels for both uniform and speckled reference beam contributions. The issues addressed in this paper have not been explored in this depth previously and they provide valuable insight into the generation of speckle noise in Laser Vibrometers.

\section{Acknowledgements}

The author wishes to acknowledge the support of the Engineering and Physical Sciences Research Council, the Leverhulme Trust and the Royal Society as well as the hospitality of the Dipartimento di Meccanica, Universita' Politecnica delle Marche, Ancona, Italy where the first part of this work was completed. 


\section{References}

1 J.D. Rigden and E.I. Gordon, “The granularity of scattered optical maser light,” Proc I.R.E 50, 2367-2368 (1962).

2 B.M. Oliver, “Sparkling spots and random diffraction,” Proc IEEE 51, 220-221 (1963).

3 R.V. Langmuir, “Scattering of laser light,” Applied Physics Letters 2(2), 29-30 (1963).

4 D. Gabor, "Laser speckle and its elimination," IBM Journal of Research and Development, 509-514 (1970).

5 J.M. Burch and J.M.J. Tokarski, "Production of multiple beam fringes from photographic scatterers,” Optica Acta 15(2), 101-111 (1968).

6 M. Francon, Laser Speckle and Applications in Optics (Academic Press, New York, 1979).

$7 \quad$ R.K. Erf (Ed.), Speckle Metrology (Academic Press, New York. 1978).

8 Y Yeh and H.Z. Cummins "Localized fluid flow measurements with an HeNe laser spectrometer,” Applied Physics Letters 4(10), 176-178 (1964).

9 D.C. Williams (Ed.), Optical Methods in Engineering Metrology (Chapman \& Hall, London, 1993).

10 J Oldengarm, A.H. Von Krieken and H. Raterink, "Laser Doppler velocimeter with optical frequency shifting,” Optics and Laser Technology 5, 249-252 (1973).

11 P. Buchave, "Laser Doppler velocimeter with variable optical frequency shift," Optics and Laser Technology 7, 11-16 (1975).

12 N.A. Halliwell, "Laser Doppler measurement of vibrating surfaces: a portable instrument,” Journal of Sound and Vibration 62, 312-315 (1979). 
13 T.A. Riener, A.C. Goding and F.E. Talke, "Measurement of head/disc spacing modulation using a two channel fiber optic laser Doppler Vibrometer," IEEE Transactions on Magnetics 24(6), 2745-2747 (1988).

14 R.W. Wlezein, D.K. Miu and V. Kibens, "Characterization of rotating flexible disks using a laser Doppler Vibrometer,” Optical Engineering 24(4), 436-442 (1984).

15 R.A. Cookson and P. Bandyopadhyay, “A fiber-optics laser Doppler probe for vibration analysis of rotating machines," Transactions of the ASME Journal of Engineering for Power 102, 607-612 (1980).

16 A.K. Reinhardt, J.R. Kadambi and R.D. Quinn, "Laser vibrometry measurements on rotating blade vibrations," Transactions of the ASME Journal of Engineering for Gas Turbines and Power 117, 484-488 (1995).

17 P. Castellini and R. Montanini, “Automotive components vibration measurements by tracking laser Doppler vibrometry: advances in signal processing,” Meas. Sci. Technol. 13 1266-1279 (2002).

18 M. Rantatalo, K. Tatar and P. Norman "Laser Doppler Vibrometry measurements of a rotating milling machine spindle,” in Proceedings of 8th International Conference on Vibrations in Rotating Machinery, (Swansea, UK, 2004), pp. 231-240.

19 A.B. Stanbridge and D.J. Ewins "Modal testing of rotating discs using a scanning LDV," in Proceedings of ASME Design Engineering Technical Conference 3(B), (Boston, USA, 1995), pp. 1207-1213.

20 N.A. Halliwell, C.J.D. Pickering and P.G. Eastwood, "The laser torsional vibrometer: a new instrument,” J. Sound and Vibration 93(4), 588-592 (1984). 
21 N.A. Halliwell and P.G. Eastwood, “The Laser Torsional Vibrometer,” Journal of Sound and Vibration 101(3), 446-448 (1985).

22 N.A. Halliwell and P.G. Eastwood, "Marine Vehicles and Offshore Installations: Laser Diagnostics of Machinery Health,” Optics and Lasers in Engineering 16(4/5), 337-350 (1992).

23 J.R. Bell and S.J. Rothberg, "Rotational vibration measurements using laser vibrometry: comprehensive theory and practical application,” Journal of Sound and Vibration 238(4), 673-690 (2000).

24 J.C. Dainty, Laser Speckle and Related Phenomena (Springer-Verlag, Berlin, 1975).

25 S.J. Rothberg, J.R. Baker and N.A. Halliwell, "Laser vibrometry: pseudo-vibrations," Journal of Sound and Vibration 135(3), 516-522 (1989).

26 L. Lading and R.V. Edwards, "Laser Velocimeters: lower limits to uncertainty,” Applied Optics 32(21), 3855-3866 (1993).

27 M. Denman, N.A. Halliwell and S.J. Rothberg, "Speckle noise reduction in laser vibrometry: experimental and numerical optimisation" in Proceedings of 2nd International Conference on Vibration Measurements by Laser Techniques, (Ancona, Italy, 1996), pp. 12-21.

28 T. Asakura and N. Takai "Dynamic laser speckles and their application to velocity measurements of the diffuse object,” Applied Physics 25, 179-194 (1981).

29 N. Takai, T. Iwai and T. Asakura, "An effect of curvature of rotating diffuse objects on the dynamics of speckle produced in the diffraction field,” Applied Physics B 26, 185-192 (1981). 
30 E. Jakeman, S.M. Watson and K.D. Ridley, "Intensity-weighted phase-derivative statistics,” J. Opt. Soc. Am. A, 18(9), 2121-2131 (2001).

31 I.D.C. Tullis, N.A. Halliwell and S.J. Rothberg, "Spatially Integrated Speckle Intensity: Maximum Resistance to Decorrelation due to In-Plane Target Displacement,” Applied Optics, 37(30), 7062-7069 (1998). 


\section{FIGURES}

Figure 1: Schematic diagram of the experimental interferometer and rotating target

Figure 2 - Speckle patterns a) By scattering from a diffuse surface b) By scattering from retroreflective tape

Figure 3 - Typical Doppler signal showing amplitude modulation

Figure 4 - Laser Vibrometer output showing significant speckle noise

Figure 5a - Variation of Doppler signal RMS and speckle noise with target-detector separation

Figure 5b - Variation of Doppler signal RMS and speckle noise with the ratio of detector size in the direction of speckle motion to speckle size, $M$

Figure 6 - Laser Vibrometer output spectrum showing pseudo-vibration

Figure 7: Variance in phase change as a function of the ratio of detector size in the direction of speckle motion to speckle size.

Figure 8 - Variation of rate of change of phase within a transition between sinusoidal components

Figure 9: $W^{2} \operatorname{Var}\left[\Delta \Phi^{\prime}\right]$ as a function of the number of partial transitions

Figure 10: Representation of the target speckle pattern matrix.

Figure 11: Simulated phase change data showing $2 \pi$ wraparounds

Figure 12: Simulated phase change data after removal of $2 \pi$ wraparounds and the effect of low

Doppler signal amplitude

Figure 13: Speckle noise prediction (uniform intensity reference beam contribution, no curvature correction). Total noise (+), Noise low-pass filtered at 5kHz (solid line).

Figure 14a: Speckle noise prediction (uniform intensity reference beam contribution). Total noise $(+)$, Total noise after track-and-hold (O), Noise low-pass filtered at 5kHz (solid line). 
Figure 14b: Speckle noise prediction (speckled reference beam contribution). Total noise (+), Total noise after track-and-hold (O), Noise low-pass filtered at 5kHz (solid line).

Figure 15: Doppler signal amplitude with uniform intensity reference beam contribution (predicted, solid line, and experimental, *) and speckled reference beam contribution (predicted, solid line, and experimental, +)

Figure 16: Comparison of predicted (solid line with * markers) and experimental $(+)$ speckle noise amplitudes (uniform intensity reference beam contribution)

Figure 17: Comparison of predicted (solid line with * markers) and experimental $(+)$ speckle noise amplitudes (speckled reference beam contribution). Dashed line shows speckle noise prediction without the effect of curvature in the target beam speckle pattern. 


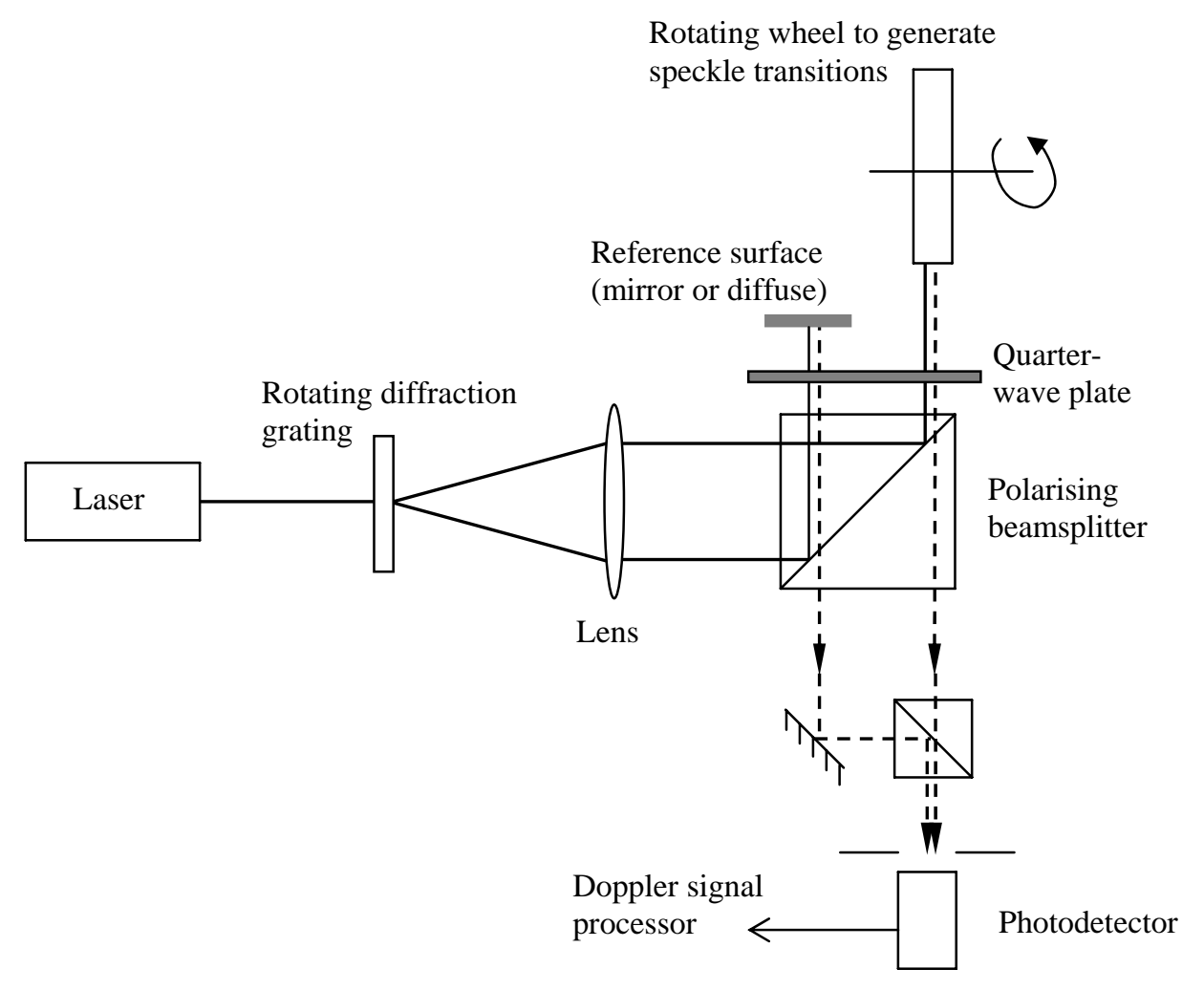

Figure 1: Schematic diagram of the experimental interferometer and rotating target 


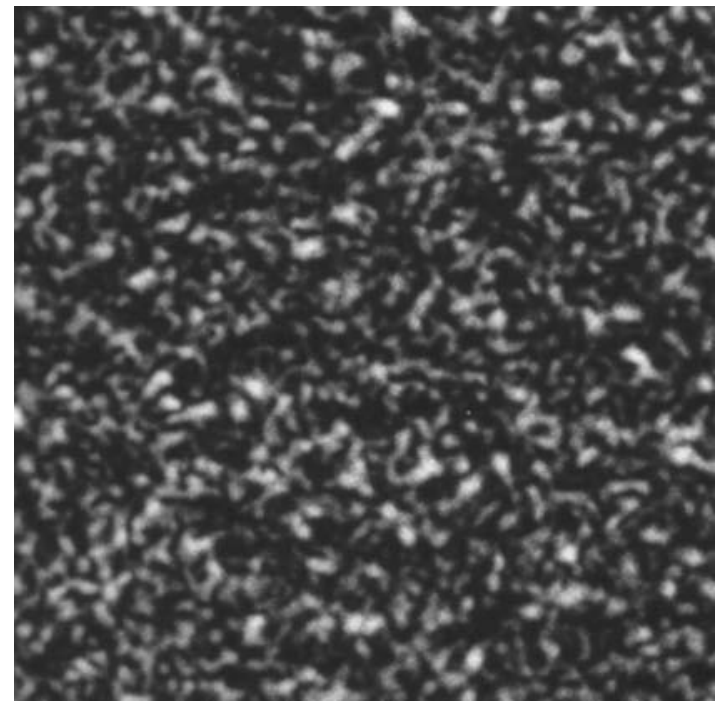

a) By scattering from a diffuse surface

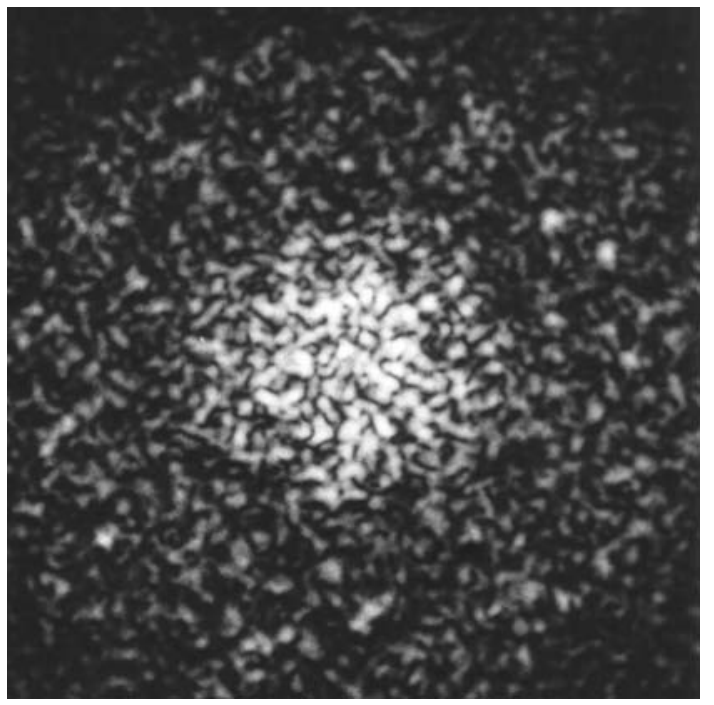

b) By scattering from retro-reflective tape

Figure 2 - Speckle patterns 


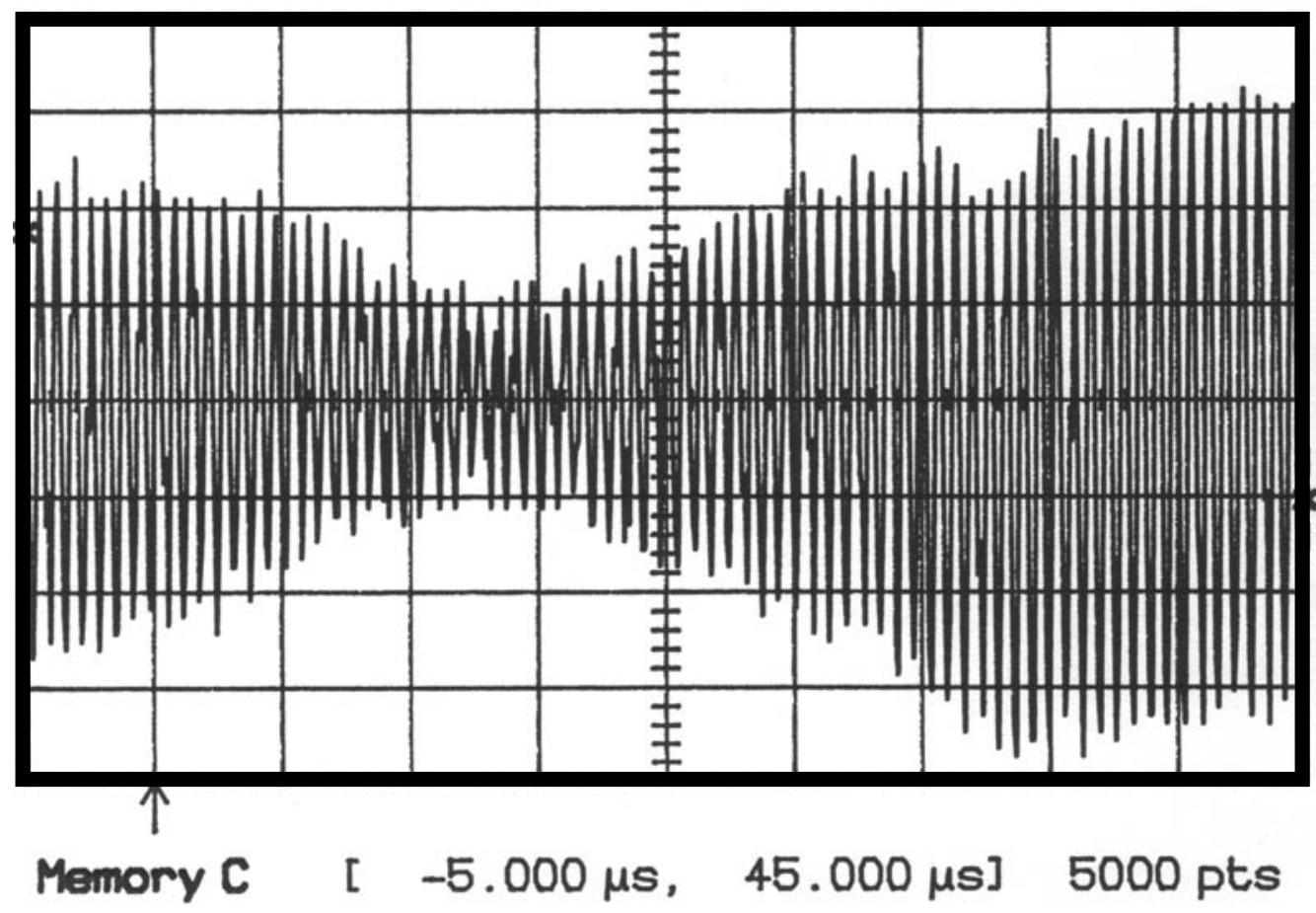

Figure 3 - Typical Doppler signal showing amplitude modulation 


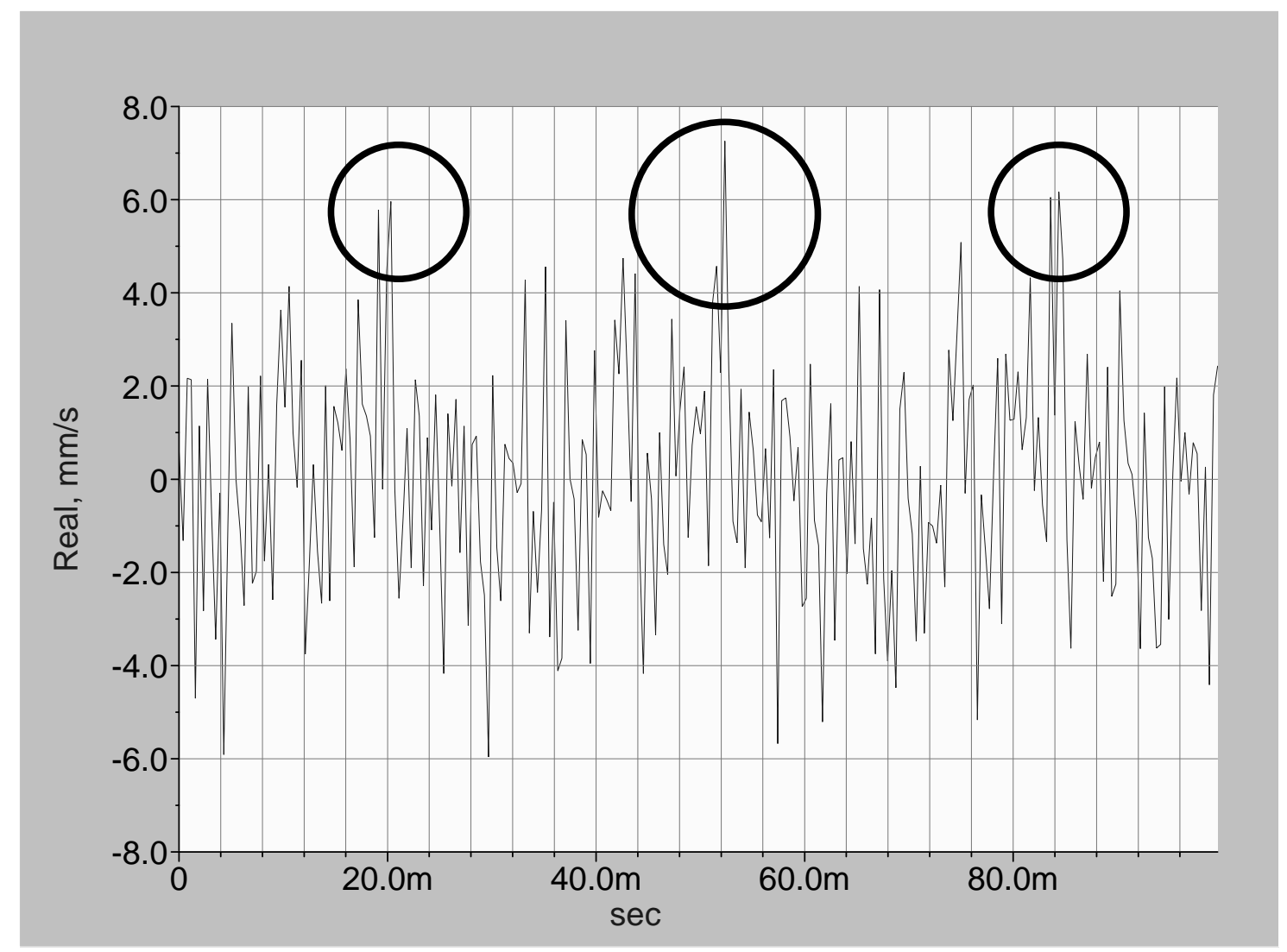

Figure 4 - Laser Vibrometer output showing significant speckle noise 


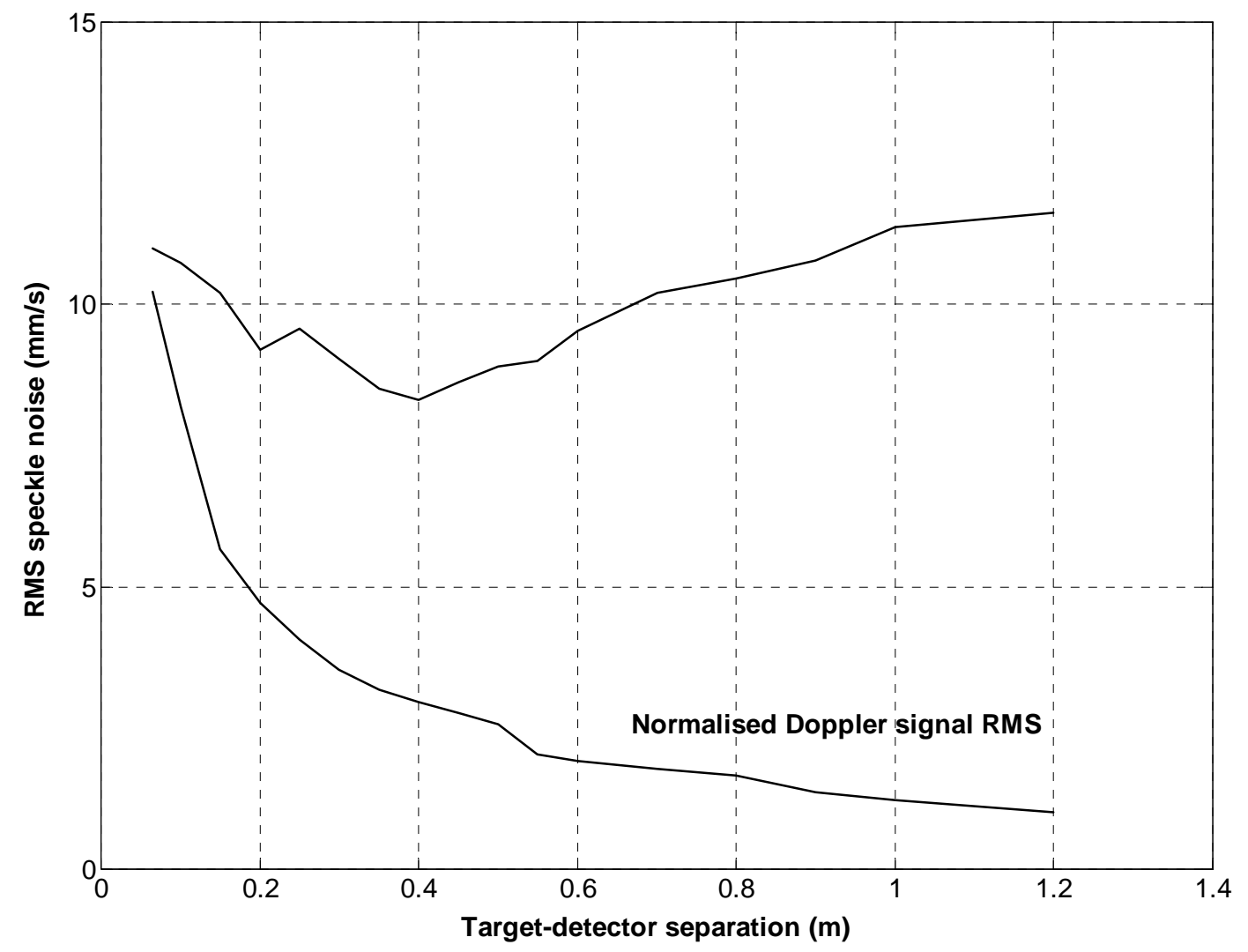

Figure 5a - Variation of Doppler signal RMS and speckle noise with target-detector separation 


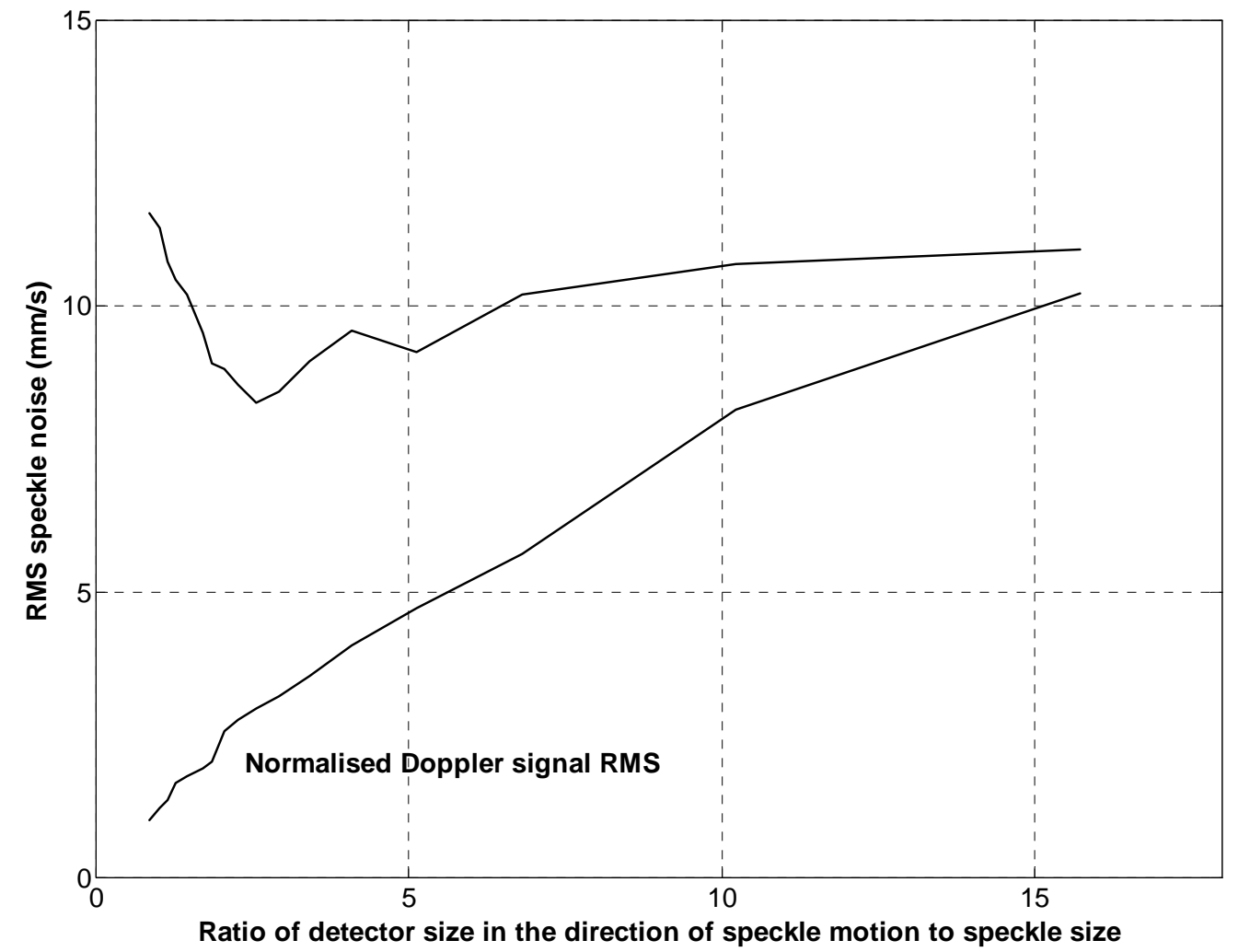

Figure 5b - Variation of Doppler signal RMS and speckle noise with the ratio of detector size in the direction of speckle motion to speckle size, $M$ 


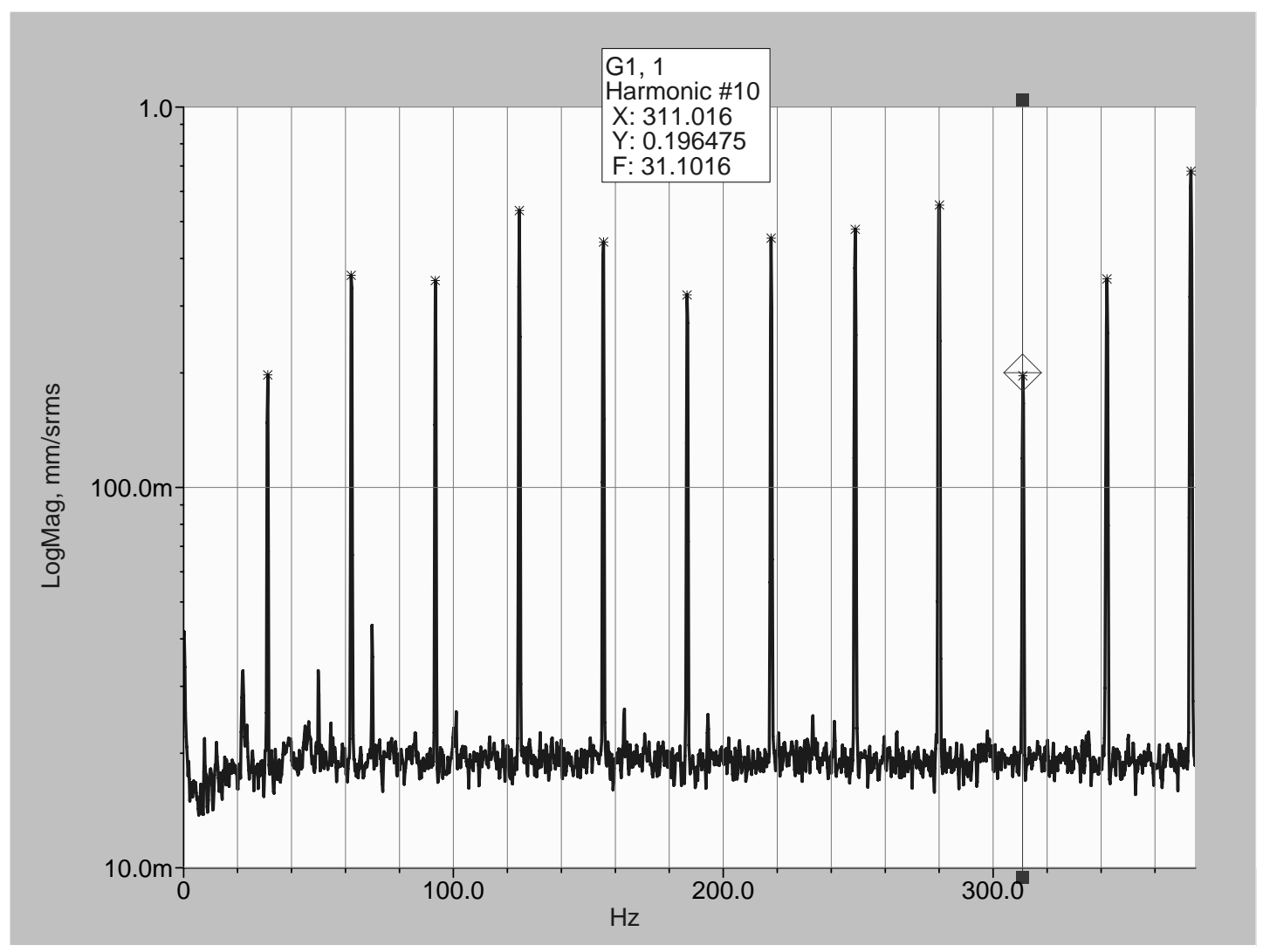

Figure 6 - Laser Vibrometer output spectrum showing pseudo-vibration 


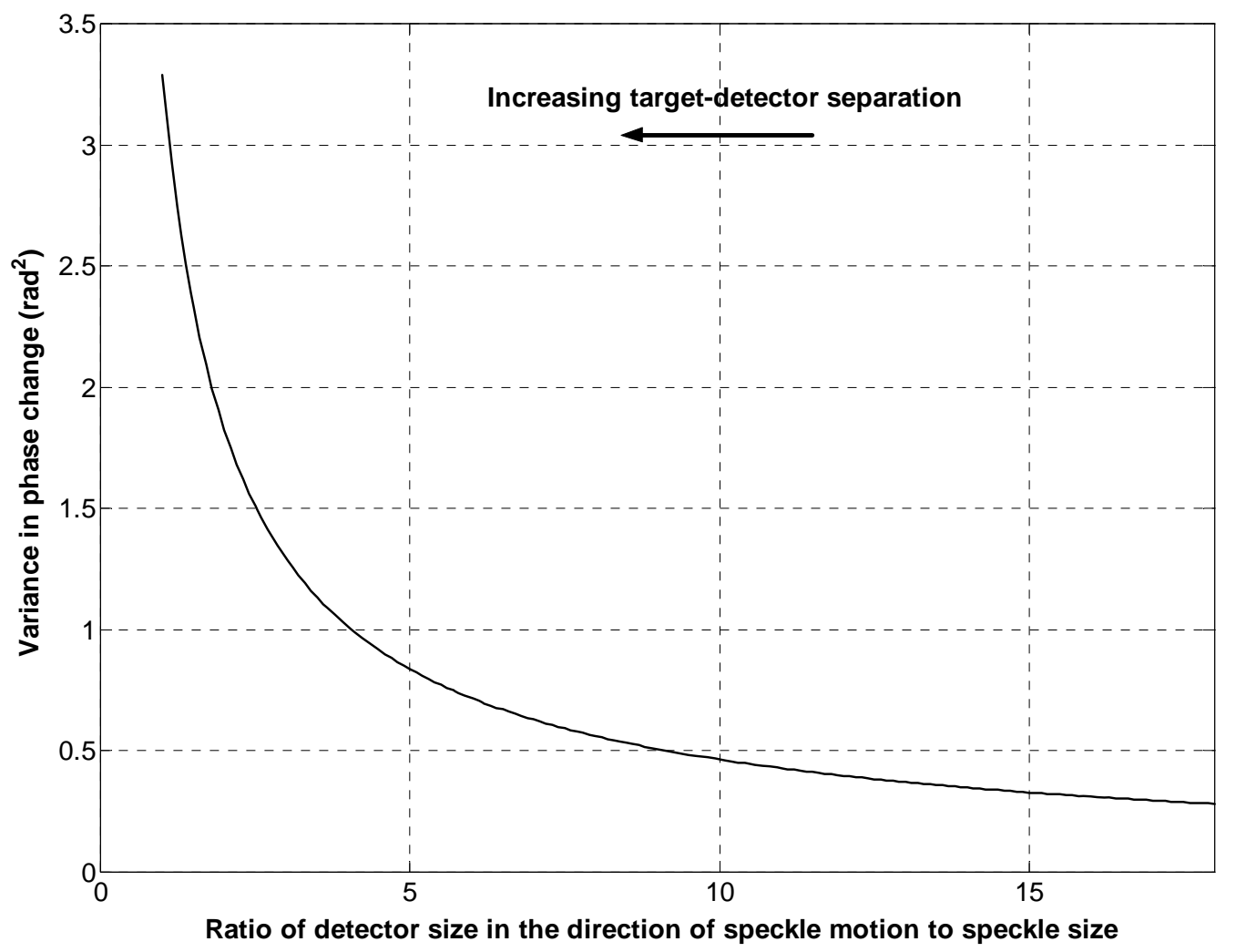

Figure 7: Variance in phase change as a function of the ratio of detector size in the direction of speckle motion to speckle size. 


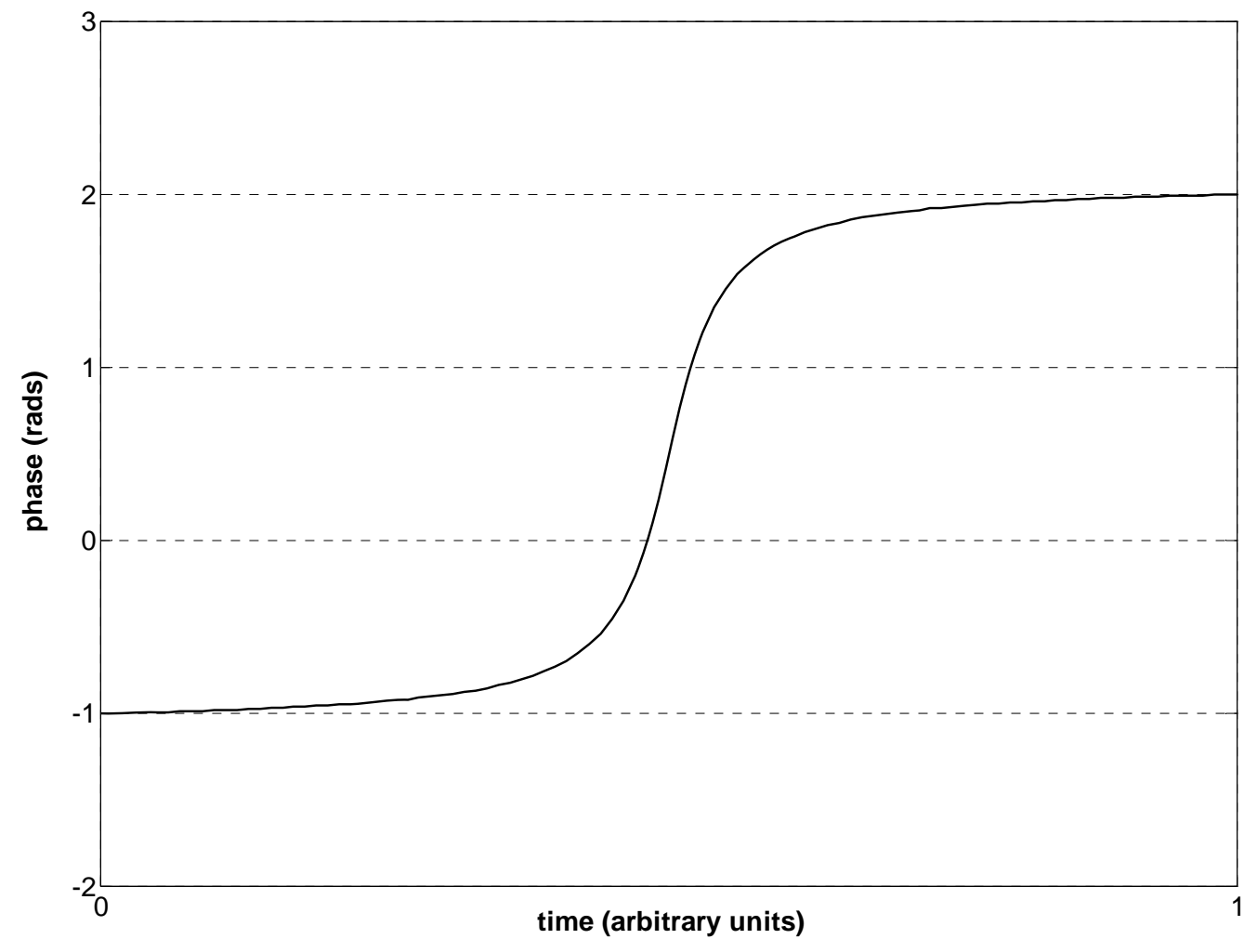

Figure 8 - Variation of rate of change of phase within a transition between sinusoidal components 


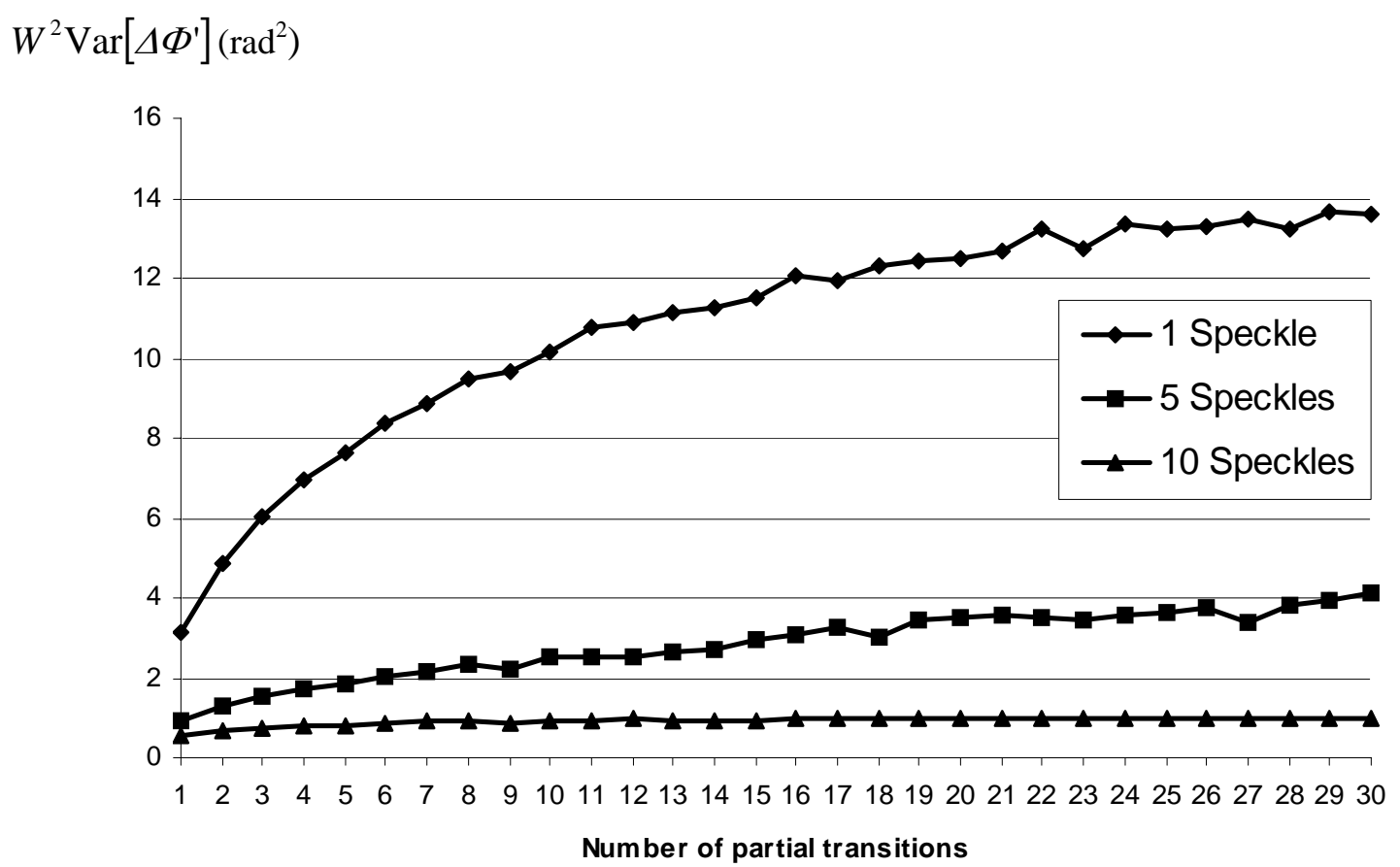

Figure 9: $W^{2} \operatorname{Var}\left[\Delta \Phi^{\prime}\right]$ as a function of the number of partial transitions 


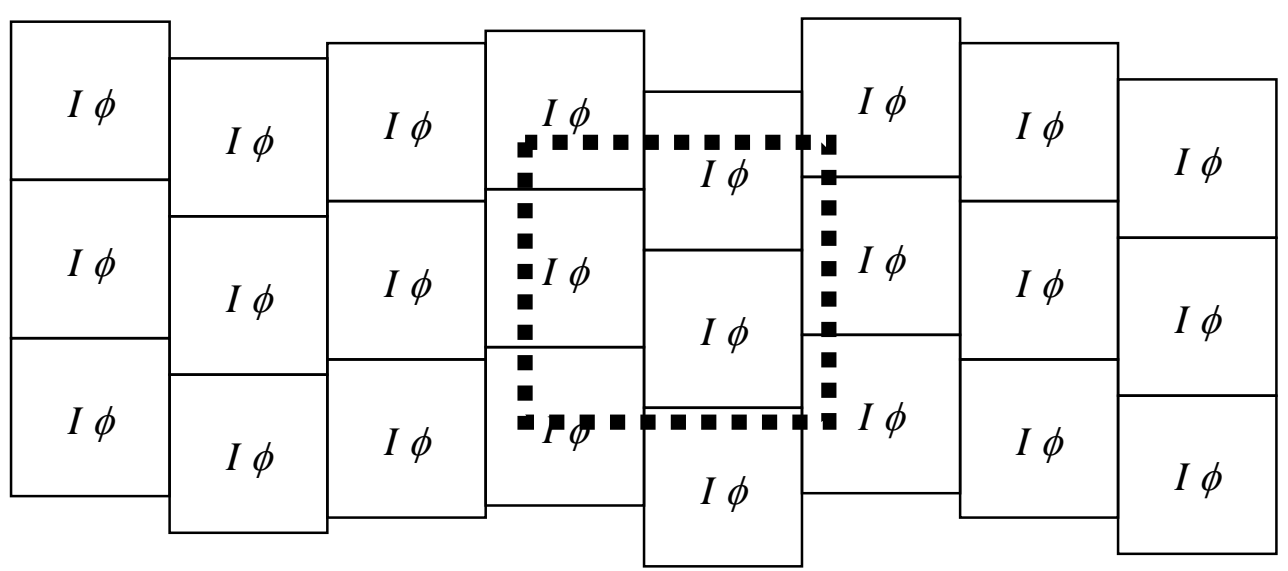

Speckle Motion

Figure 10: Representation of the target speckle pattern matrix. 


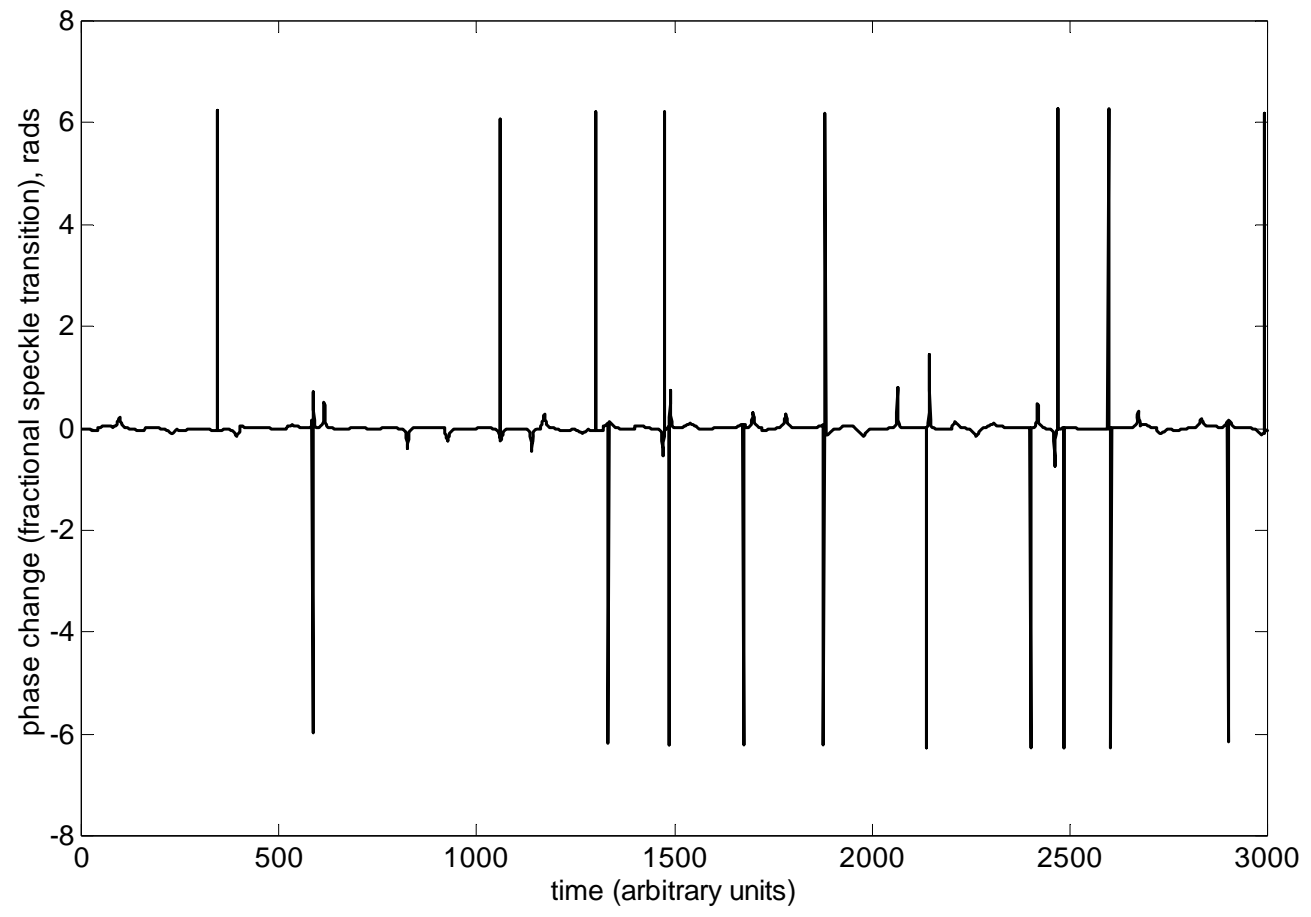

Figure 11: Simulated phase change data showing $2 \pi$ wraparounds 

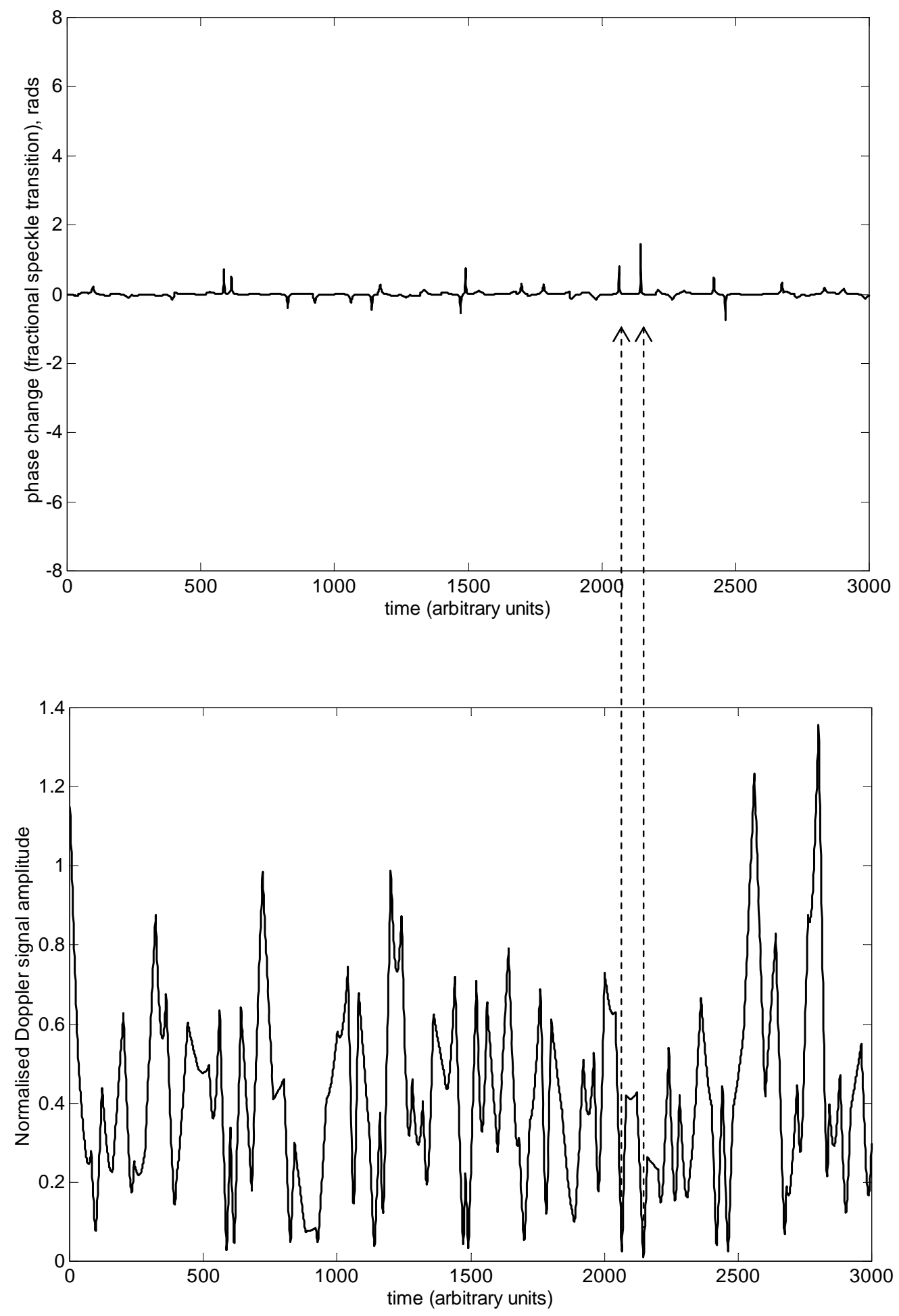

Figure 12: Simulated phase change data after removal of $2 \pi$ wraparounds and the effect of low Doppler signal amplitude 


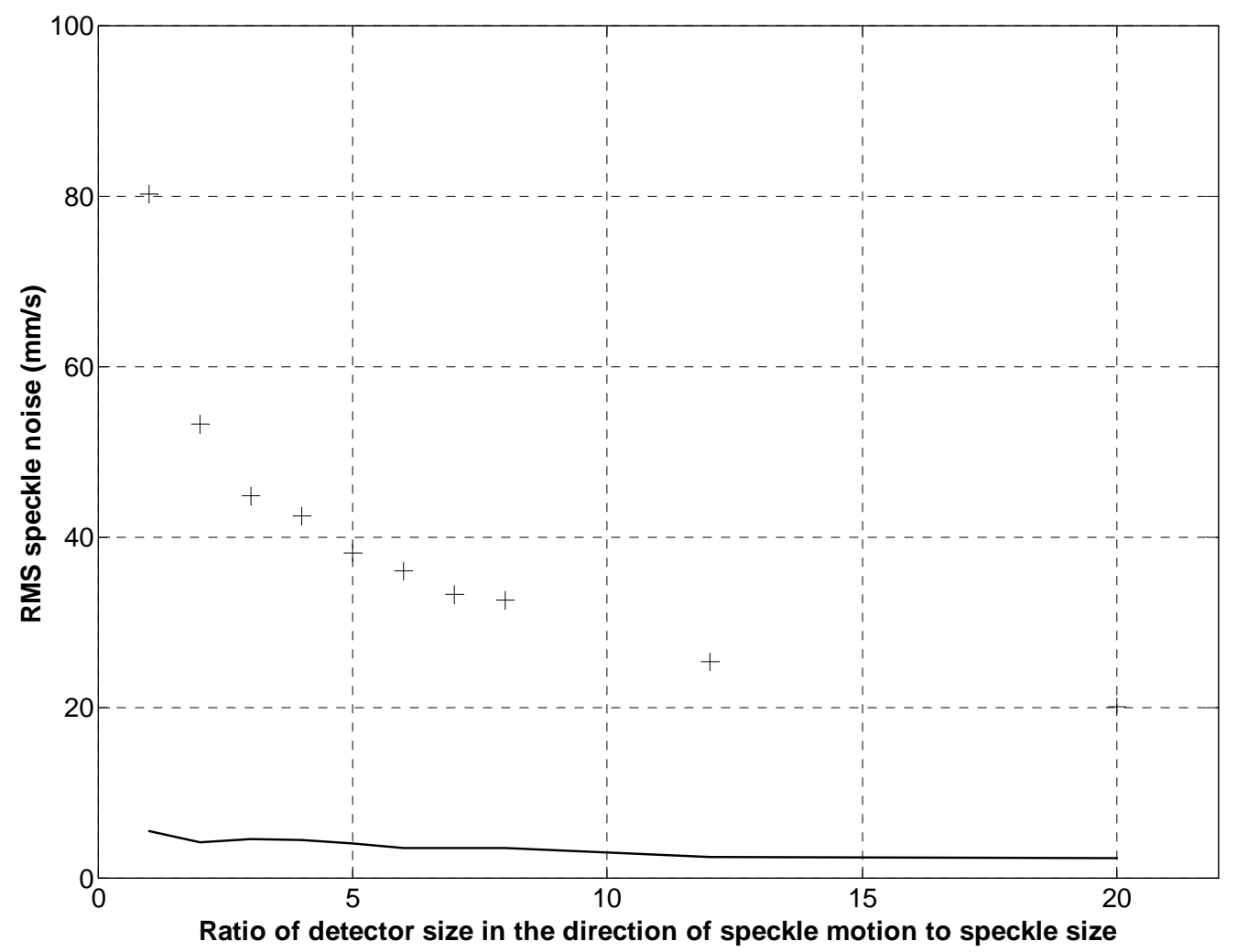

Figure 13: Speckle noise prediction (uniform intensity reference beam contribution, no curvature correction). Total noise (+), Noise low-pass filtered at $5 \mathrm{kHz}$ (solid line). 


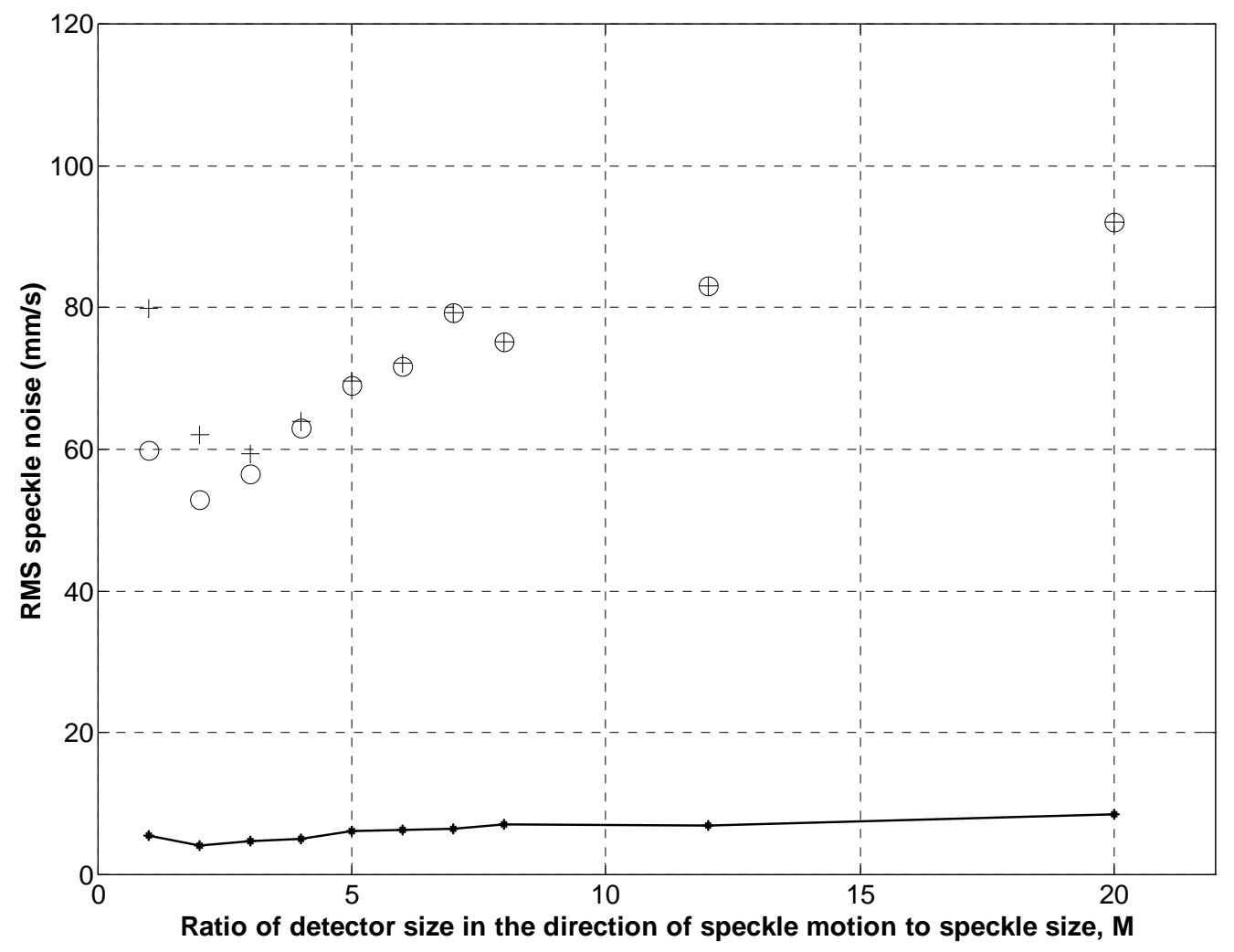

Figure 14a: Speckle noise prediction (uniform intensity reference beam contribution). Total noise $(+)$, Total noise after track-and-hold (O), Noise low-pass filtered at 5kHz (solid line). 


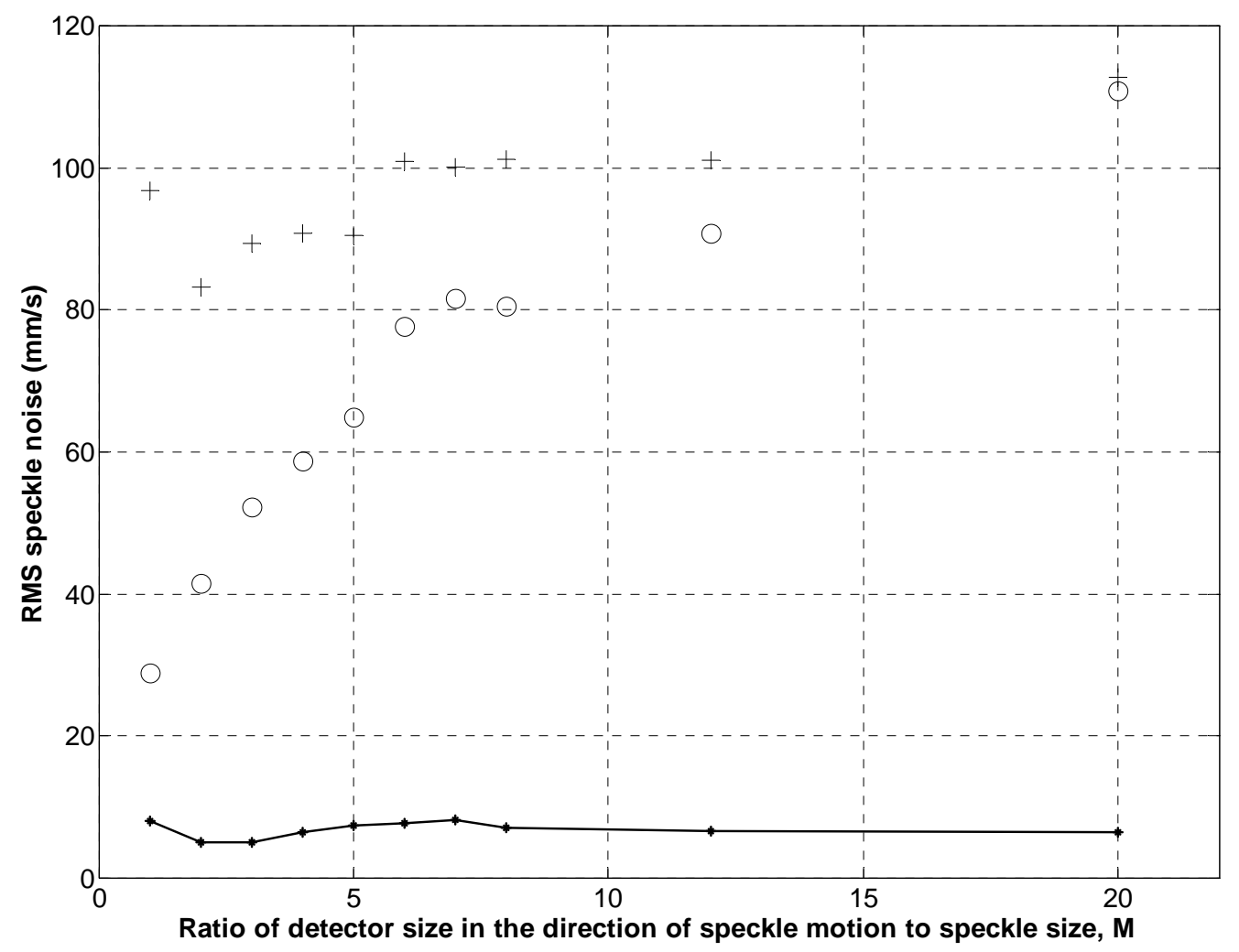

Figure 14b: Speckle noise prediction (speckled reference beam contribution). Total noise $\left(^{+}\right)$, Total noise after track-and-hold (O), Noise low-pass filtered at $5 \mathrm{kHz}$ (solid line). 


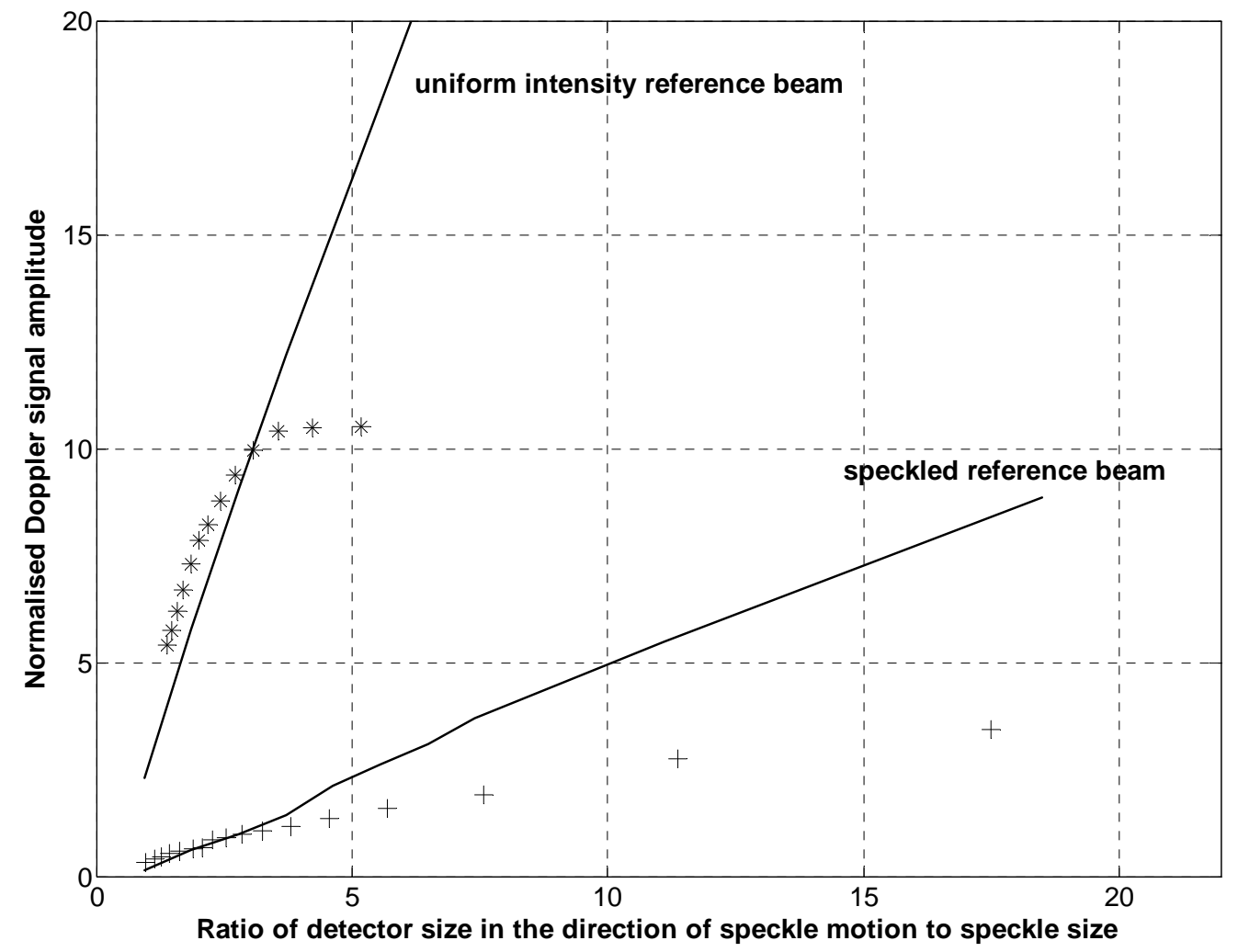

Figure 15: Doppler signal amplitude with uniform intensity reference beam contribution (predicted, solid line, and experimental, *) and speckled reference beam contribution (predicted, solid line, and experimental, +) 


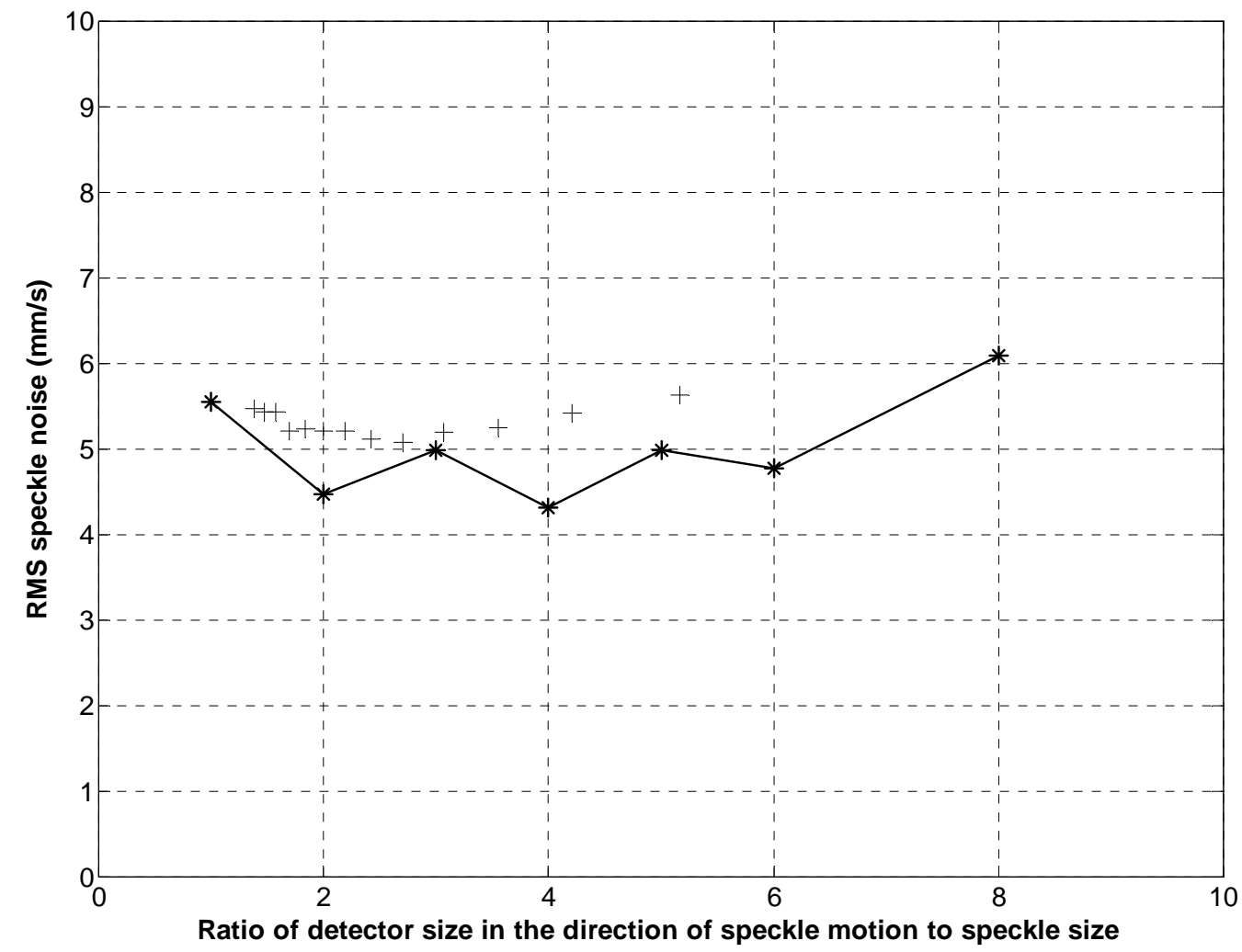

Figure 16: Comparison of predicted (solid line with * markers) and experimental (+) speckle noise amplitudes (uniform intensity reference beam contribution) 


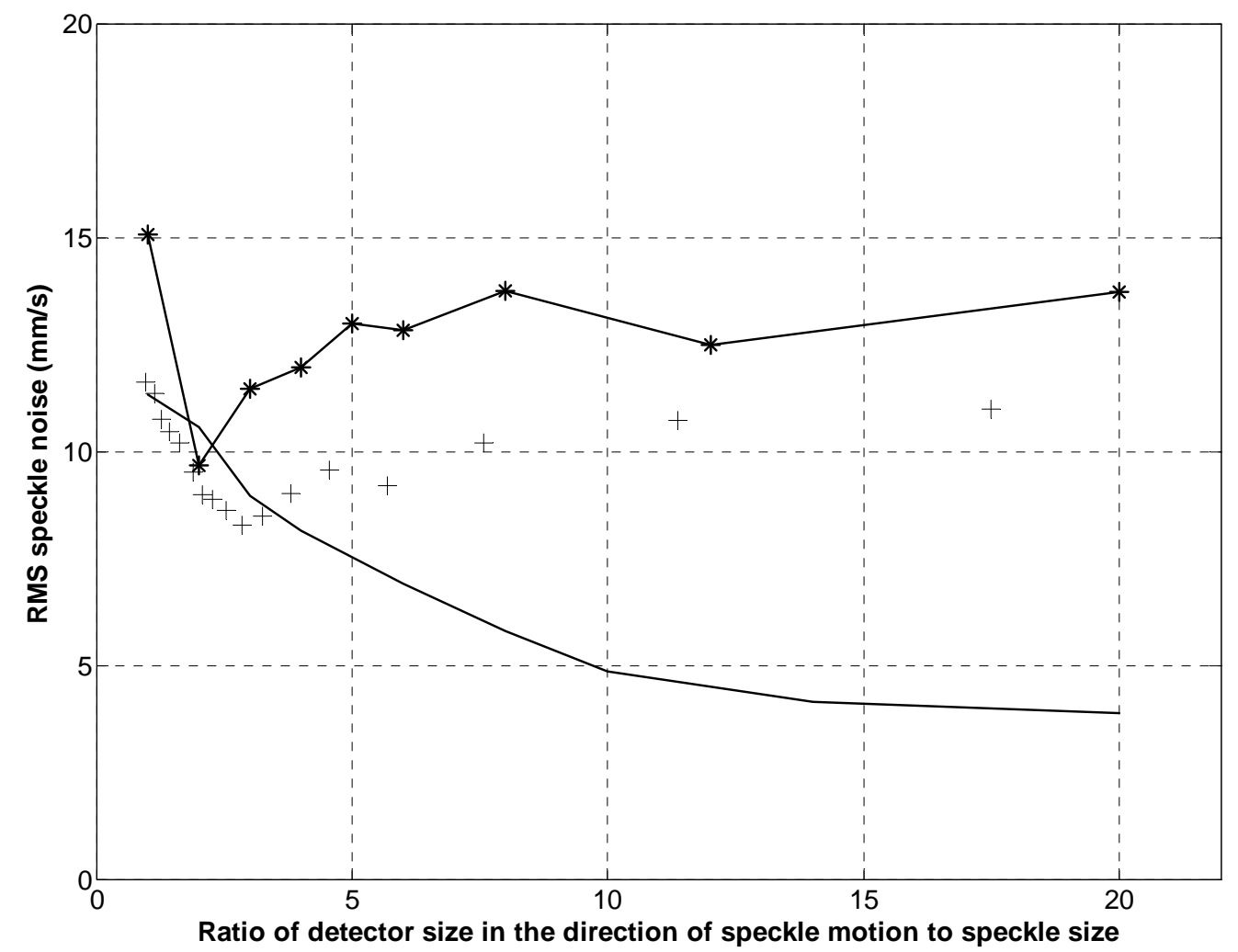

Figure 17: Comparison of predicted (solid line with * markers) and experimental $(+)$ speckle noise amplitudes (speckled reference beam contribution). Dashed line shows speckle noise prediction without the effect of curvature in the target beam speckle pattern 